\title{
Compact coalgebras, compact quantum groups and the positive antipode
}

\author{
Andrés Abella ${ }^{1}$ \\ Facultad de Ciencias, Universidad de la República, Iguá 4225, 11400 Montevideo, \\ Uruguay \\ E-mail address: andres@cmat.edu.uy

\section{Walter Ferrer Santos ${ }^{2}$} \\ Facultad de Ciencias, Universidad de la República, Iguá 4225, 11400 Montevideo, \\ Uruguay \\ E-mail address: wrferrer@cmat.edu.uy
}

\section{Mariana Haim ${ }^{3}$}

Facultad de Ciencias, Universidad de la República, Iguá 4225, 11400 Montevideo, Uruguay

E-mail address: negra@cmat.edu.uy

\begin{abstract}
In this article -that has also the intention to survey some known results in the theory of compact quantum groups using methods different from the standard and with a strong algebraic flavor- we consider compact o-coalgebras and Hopf algebras. In the case of a ०-Hopf algebra we present a proof of the characterization of the compactness in terms of the existence of a positive definite integral, and use our methods to give an elementary proof of the uniqueness - up to conjugation by an automorphism of Hopf algebras- of the compact involution appearing in [4]. We study the basic properties of the positive square root of the antipode square that is a Hopf algebra automorphism that we call the positive antipode. We use it - as well as the unitary antipode and Nakayama automorphism- in order to enhance our understanding of the antipode itself.
\end{abstract}

\footnotetext{
${ }^{1}$ The author would like to thank Conycit-MEC, Uruguay.

2 The author would like to thank Csic-UDELAR, Conycit-MEC, Uruguay.

3 The author would like to thank PEDECIBA, MEC-Udelar, Uruguay.
} 


\section{Introduction}

The family of compact groups plays an essential role in the theory of group representations. This is clearly illustrated in the pioneering work of A. Hurwitz and I. Schur: see for example Hurwitz's "landmark paper" [11] (c.f. Borel's [7, Chapter 2, Section 2]) or the later paper by I. Schur [19].

A specially illustrative example is the unitäre Beschränkung -later called unitarian trick- that $\mathrm{H}$. Weyl used in order to prove the complete reducibility of the representations of a semisimple group -see [23, 24, 25]-.

Since the mid 1980's it has been established as a paradigm that the group objects in non commutative geometry, i.e. the so called quantum groups are the -non necessarily commutative- Hopf algebras.

Thus, after the above notion of quantum group was introduced, it was natural to expect the development of an adequate concept of compact quantum group.

This concept was introduced in Woronowicz's seminal work -see [27]where the basic structure was defined in terms of $\mathrm{a} \star$-operation on a general Hopf algebra.

Later, the main algebraic structure underlying the definition of compact quantum group was introduced -and called a $C Q G$ algebra- where, following the same spirit than in Woronowicz's paper, there is also a compatible *-operation that plays the central foundational role. See for example the paper [3] and the later [8] with a more formal presentation. In book form, the reference [12] has rather complete a treatment of compact quantum groups and their representations.

In the present work we use the approach to the concept of compact quantum group introduced in [1], [2], and [15], where instead of the $\star$-operation the equivalent concept of a o-structure is taken as the fundamental notion. This approach is more convenient for our purposes. In particular it allows the early introduction of the basic concept of compact coalgebra.

In the following we present a brief description of the contents of this paper.

In Section 2 we recall a few basic notations and well known results in coalgebra and comodule theory that will be used throughout the paper.

In Section 3 we recall the definition of o-coalgebra and show that the existence of a 0 -structure on a coalgebra $C$ is intimately related with the existence of the analog of a duality functor in the category of $C$-comodules. Then we introduce the notion of compact o-coalgebra in terms of the existence of unitary inner products in the objects of the category of $C$ comodules. In particular, this definition shows that compact o-coalgebras are cosemisimple. 
In Section 4 we give different characterizations of the notion of compact o-coalgebras in terms of unitary inner products and also of positive definite Fourier forms and present a structure theorem that refines the corresponding well known result for cosemisimple coalgebras -these results appear in Theorem 4.3-.

In Section 5 we specialize to the situation of Hopf algebras whose underlying coalgebra and algebra structures have a compatible o-operator. In Theorem 5.5, we prove the following basic positivity result: if $V$ and $V^{*}$ admit unitary inner products, there exists a positive isomorphism between $V$ and $V_{S^{2}}$-where $V_{S^{2}}$ denotes de $\mathbb{C}$-space $V$ with the structure corestricted with the automorphism $S^{2}$.

In Section 6 we define in terms of a o-structure, the concept of compact quantum group and present an intrinsic proof in Theorem 6.9 -see also Theorem 6.7 - of the characterization of the compactness in terms of the positivity of the bilinear product defined from the integral -the Haar measure in Woronowicz's nomenclature-- This proof is based upon the existence -for every $H$-comodule $V$ - of a positive isomorphism between $V$ and $V_{S^{2}}$ mentioned before.

Section 7 is dedicated to the presentation of an intrinsic and elementary algebraic proof of a result due to Andruskiewitsch that appeared in [4], and that guarantees the uniqueness up to Hopf algebra automorphisms, of the compact involution, i,e., of the o-structure in the given Hopf algebra.

In Section 8 we begin the study of some of the basic properties of the antipode in a compact quantum group. We show that for a compact quantum group, there is a positive automorphism of $H$ that we call the positive antipode -denoted as $S_{+}$- that when squared coincides with the automorphism $S^{2}$. Then, we use some of the standard tools in coFrobenius Hopf algebra theory -Radford's formula, Nakayama automorphism, the modular function, etc.- in order to describe some of the basic properties of $S$ and of $S_{+}$. We show that $S_{+}$is given by conjugation by a positive multiplicative functional $\beta$-Theorem 8.7 and Corollary $8.11-$. We show that the Nakayama automorphism is a positive algebra automorphism and define its postive square root $\mathcal{P}$, that we write in terms of $\beta$. We compute explicitly the adjoint of $S$ and show that the antipode is a normal operator with respect to the inner product given by the integral, if and only if the positive antipode is the identity and this happens if and only if $S^{2}=$ id -Theorem $8.15-$. We also compute explicitly the so called unitary antipode in terms of $S, S_{+}$and of the Nakayama automorphism.

We finish the paper with a short section -Section 9- where we illustrate the methods developed, in the case of the compact quantum group $\mathrm{SU}_{\mu}(2, \mathbb{C})$. In particular we compute explicitly the positive antipode $S_{+}$ and the unitary antipode $U$. 
The authors would like to thank Nicolás Andruskiewitsch who -besides introducing them to this subject- shared many of his ideas, insights and widespread knowledge.

We also would like to thank the referee for her/his very useful comments and corrections, that we are sure made the paper more useful.

\section{Preliminaries and basic notations}

In this section we fix the notations and recall a few basic definitions and concepts in coalgebra theory. See $[1,13]$ and $[22]$ for more details on some of the needed prerequisites and $[18,21]$ for general background in Hopf algebra theory.

All the objects are defined over $\mathbb{C}$ and the category of $\mathbb{C}$-vector spaces will be denoted sometimes by $\mathcal{V}$. If $V$ is a $\mathbb{C}$-vector space and $A: V \rightarrow W$ is a linear map, then $V_{c}$ is the conjugate of $V$ and $A_{c}: V_{c} \rightarrow W_{c}$ the conjugate linear map. Moreover, $V_{c}^{*}$ will denote $\left(V^{*}\right)_{c}$.

For coalgebras and comodules we use Sweedler's notation. The symbols: $\mathcal{M}^{C}$ and ${ }^{C} \mathcal{M}$ represent the categories of right and left $C$-comodules respectively and $\mathcal{M}_{f}^{C}$ and ${ }^{C} \mathcal{M}_{f}$ respectively are the full categories of finite dimensional objects.

Definition 2.1. Let $V$ be a finite dimensional vector space and $\mathcal{B}=$ $\left\{e_{1}, \cdots, e_{n}\right\}$ a basis. Recall the definition of the coalgebra $c(V)=\left(V^{*} \otimes\right.$ $V, \Delta, \varepsilon): \Delta(\alpha \otimes v)=\sum \alpha \otimes e_{i} \otimes e^{i} \otimes v$, and $\varepsilon: V^{*} \otimes V \rightarrow \mathbb{k}$ is $\varepsilon(\alpha \otimes v)=\alpha(v)$ -above $\mathcal{B}^{*}=\left\{e^{1}, \cdots, e^{n}\right\}$ is the dual basis of $\mathcal{B}^{-}$. The space $V$ is a right $c(V)$-comodule via $\chi_{0}: V \rightarrow V \otimes c(V)$, given by $v \mapsto \sum e_{i} \otimes e^{i} \otimes v$. Then, $c(V)$ is a simple coalgebra and $\left(V, \chi_{0}\right)$ is an irreducible right $c(V)$-comodule (see for example [1, Section 3]).

Observation 2.2. (1) It is equivalent to give a right $C$-comodule $(V, \chi) \in \mathcal{M}_{f}^{C}$ and to give a morphism of coalgebras $c_{\chi}: c(V) \rightarrow C$. The morphism of coalgebras is the map $c_{\chi}(\alpha \otimes v)=\sum \alpha\left(v_{0}\right) v_{1}$. Notice that in the case that $V$ is a not necessarily finite dimensional $C$-comodule, we can still define the linear map $c_{\chi}: V^{*} \otimes V \rightarrow C$ but the vector space $V^{*} \otimes V$ is not endowed with a natural coalgebra structure but $c_{\chi}\left(V^{*} \otimes V\right)=\operatorname{Coeff}(V) \subset C$ is a subcoalgebra of $C$, called the subcoalgebra of coefficients of $V$. In that situation the $C$-comodule structure of $V$ is induced from a Coeff $(V)$-comodule structure. In other words, $\chi: V \rightarrow V \otimes C$ can be split as $V \stackrel{\chi}{\rightarrow}$ $V \otimes \operatorname{Coeff}(V) \subset V \otimes C$. In this situation the relationship between the $C$-comodule structure of $V$ and the maps $\chi_{0}$ and $c_{\chi}$ introduced 
respectively in Definition 2.1 and Observation 2.2 part (1), is given by the commutativity of the diagram below.

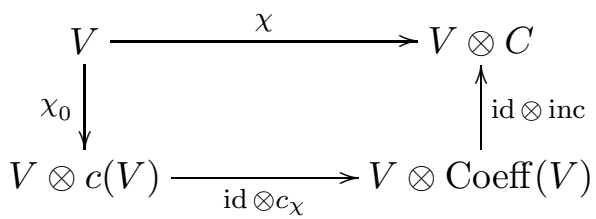

(2) In the above notations, given $(V, \chi) \in \mathcal{M}_{f}^{C}$, we define the matrix $T \in \mathrm{M}_{\mathrm{n}}(C)$, as $T=\left(c_{\chi}\left(e^{j} \otimes e_{i}\right)\right)_{1 \leq i, j \leq n}$. The matricial coefficients of the $C$-comodule $V$, i.e. the entries of $T$, are defined by the formulæ $\chi\left(e_{j}\right)=\sum_{i} e_{i} \otimes t_{i j}$. Clearly, $\Delta\left(t_{i j}\right)=\sum_{k} t_{i k} \otimes t_{k j} ; \varepsilon\left(t_{i j}\right)=\delta_{i j}$. It is well known that $V$ is irreducible if and only if the matrix coefficients $\left\{t_{i j}\right\}$ are linearly independent.

In the context of linear actions of groups, the algebra of coefficients is usually called the algebra of representative functions.

We list a few results concerning the above construction. Most of the proofs can be found in [1, Section 4].

Observation 2.3. (1) Assume that $I \subset C$ is a right coideal with the property that $\operatorname{Coeff}(I) \subset I$. Then $\Delta(I) \subset I \otimes \operatorname{Coeff}(I)$ and applying $\varepsilon \otimes$ id we deduce that $I \subset \operatorname{Coeff}(I)$. Hence $I=\operatorname{Coeff}(I)$ is a subcoalgebra.

(2) If $V \in \mathcal{M}^{C}$ is irreducible, then $\operatorname{Coeff}(V)$ is a simple subcoalgebra of $C$ and conversely if $C$ is a simple coalgebra, there exists an irreducible $C$-comodule $V$ such that $C=\operatorname{Coeff}(V) \cong c(V)$. Moreover if $C$ is a simple and $W$ is an arbitrary non zero $C$-comodule, then Coeff $(W)=C$.

(3) Let $C$ be an arbitrary coalgebra and $C_{1}=\operatorname{Coeff}\left(V_{1}\right), C_{2}=$ Coeff $\left(V_{2}\right) \subset C$ be simple subcoalgebras with $V_{1}, V_{2}$ irreducible $C$ comodules, then $C_{1}=C_{2}$ if and only if $V_{1} \cong V_{2}$ as $C$-comodules.

(4) If $C$ can be decomposed into a sum of irreducible $C$-comodules: $C=\bigoplus\left\{V_{\rho}: \rho \in \mathcal{R}\right\}$, then an arbitrary $C$-comodule $V$, is isomorphic to $V_{\rho}$ for some $\rho \in \mathcal{R}$ and any simple subcoalgebra $D \subset C$ is of the form $D=\operatorname{Coeff}\left(V_{\rho}\right)$ for some $\rho \in \mathcal{R}$.

Observation 2.4. A hermitian form $\beta: V \otimes V_{c} \rightarrow \mathbb{C}$ defined on $V$ induces a linear morphism $\psi_{\beta}: V \rightarrow V_{c}^{*}$ defined as $\psi_{\beta}(u)(v)=\beta(v, u)$.

Assume that $V$ and $V^{*}$ are finite dimensional and endowed with inner products $\beta: V \otimes V_{c} \rightarrow \mathbb{C}$ and $\gamma: V^{*} \otimes V_{c}^{*} \rightarrow \mathbb{C}$. Next, consider the corresponding isomorphisms $\psi_{\beta}: V \rightarrow V_{c}^{*}$ and $\psi_{\gamma}: V^{*} \rightarrow V_{c}^{* *}$ and the linear map $\phi: V \rightarrow V$ defined by the commutativity of the following 
diagram:

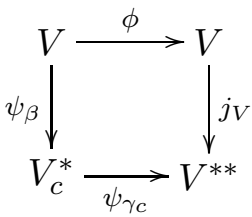

where $j_{V}: V \rightarrow V^{* *}$ denotes the canonical isomorphism, i.e. $\phi$ is defined by the equality: $\gamma\left(\psi_{\beta}(v), \psi_{\beta}(u)\right)=\beta(\phi(u), v), \forall u, v \in V$.

Then $\beta(\phi(v), v)>0$, for all $0 \neq v \in V$, i.e. $\phi: V \rightarrow V$ is $\beta$-positive definite.

Some aspects of the theory of o-coalgebras, unitary comodules and compactness - that will be treated later- can be viewed with profit in terms of Fourier forms and Fourier products in the coalgebra. The theory of Fourier forms and Fourier products for general coalgebras was developped in [9], we recall here a few basic definitions, the fact that the basic field is $\mathbb{C}$ is not relevant here.

Definition 2.5. (1) A Fourier form in $C$ is a bilinear map $\omega: C \otimes$ $C \rightarrow \mathbb{C}$ with the property that for all $c, d \in C, \sum \omega\left(c, d_{1}\right) d_{2}=$ $\sum c_{1} \omega\left(c_{2}, d\right)$. The Fourier form is said to be normal if for all $c \in C$, $\sum \omega\left(c_{1}, c_{2}\right)=\varepsilon(c)$. The Fourier form is symmetric if $\omega(c, d)=$ $\omega(d, c)$ for all $c, d \in C$.

(2) A Fourier product in $C$ is a bilinear map $\star: C \otimes C \rightarrow C$ with the property that for all $c, d \in C, \Delta(c \star d)=\sum\left(c \star d_{1}\right) \otimes d_{2}=$ $\sum c_{1} \otimes\left(c_{2} \star d\right)$.

(3) If $H$ is a Hopf algebra and $\varphi: H \rightarrow \mathbb{C}$ is a right integral ${ }^{1}$ on $H$ i.e. a linear map such that $\sum \varphi\left(x_{1}\right) x_{2}=\varphi(x) 1$ for all $x \in H$, then $\omega_{\varphi}(x, y)=\varphi(S(x) y)$ is a Fourier form -see [9]-. Moreover, the integral $\varphi$ is normal -i.e. it satisfies that $\varphi(1)=1$ - if and only if $\omega_{\varphi}$ is a normal Fourier form. Conversely, if $\omega$ is a Fourier form, then the linear map $x \mapsto \omega(1, x): H \rightarrow \mathbb{C}$ is a right integral on $H$.

Below we establish a bijective correspondence between Fourier forms and Fourier products on a coalgebra $C$. Indeed: $\omega \mapsto \star_{\omega}, \star \mapsto \omega_{\star}$, where for all $c, d \in C$,

$$
c \star_{\omega} d=\sum \omega\left(c, d_{1}\right) d_{2}=\sum c_{1} \omega\left(c_{2}, d\right), \omega_{\star}(c, d)=\varepsilon(c \star d),
$$

\footnotetext{
${ }^{1}$ In a first version of this paper and also in some previous papers by the authors - see for example [9]- the name cointegral was used for this kind of maps. We changed it following the referee's suggestion. The present name is more compatible with the usual nomenclature on the subject.
} 
It is clear that the Fourier form $\omega$ is normal if and only if the associated Fourier product satisfies the following condition: $\sum x_{1} \star_{\omega} x_{2}=x$.

The following properties are easy to verify.

Observation 2.6. (1) If $\star_{1}$ and $\star_{2}$ are arbitrary Fourier products in $C$, then $x \star_{1}\left(y \star_{2} z\right)=\left(x \star_{1} y\right) \star_{2} z$.

(2) If $\star$ is a Fourier product in $C$ and $L, R$ are respectively a left and a right coideal in $C$, then $R \star L \subseteq R \cap L$. Hence, if $D$ and $E$ are two different simple subcoalgebras of $C$, then $D \star E=0$ and therefore $\omega_{\star}(D \otimes E)=0$.

(3) If $C$ is cosemisimple and $\omega: C \otimes C \rightarrow \mathbb{C}$ is a right non degenerate Fourier form, then it follows from the above that for every simple subcoalgebra $D \subseteq C$, the restricted form $\omega: D \otimes D \rightarrow \mathbb{C}$ is right (and left -since $\bar{D}$ is finite dimensional-) non degenerate.

(4) If $D$ is a finite dimensional coalgebra and $\omega, \star$ are a form and a product associated to each other as above, then: $\omega: D \otimes D \rightarrow \mathbb{C}$ is right (and left) non degenerate if and only if $\star$ has a neutral element $s \in D$. Explicitely, $s$ is the only element in $D$ that verifies $\omega(c, s)=\varepsilon(c)=\omega(s, c), \forall c \in D$.

\section{Compact o-coalgebras}

If $G$ is a compact topological group and $\mu$ is an invariant measure, a standard tool in the representation theory of $G$ consists in "unitarizing" all the $G$-modules. Given an arbitrary inner product in a $G$-module $V$, by integration we define on $V$ a $G$-invariant inner product and in that manner we can assume that the action of the group on $V$ is given by isometries.

In order to generalize the theory of compact groups to general coalgebras, it is natural to assume as the starting point of the abstract definition of compactness of $C$, the existence of an inner product on every comodule satisfying a condition of $C$-invariance - see more precisely Definition 3.9-.

This will lead immediately to the concept of compact o-coalgebra that is presented in this section.

The notion of a o-structure in the context of Hopf algebras was originally defined in [15], where the concept was presented in terms of a $\star$-structure and of the antipode.

Later the definition of a o-structure in the more general set up of coalgebra theory appeared in [2] (see also [1] for a more recent and detailed presentation).

The algebra dual to a o-coalgebra is a $\star$-algebra, where the operator $\star$ is the dualization of $\circ$. Many concepts and proofs in the realm of o-coalgebras are counterparts of concepts and proofs for $\star$-algebras. 
Definition 3.1. (1) Assume that $(C, \Delta, \varepsilon)$ is a coalgebra over the field of complex numbers. A o-structure on $C$ is a linear map o : $C \rightarrow C_{c}$ that is involutive and anticomultiplicative. The pair $(C, \circ)$ is called a o-coalgebra.

(2) If $(C, \circ)$ and $(D, \diamond)$ are two o-coalgebras, a morphism of o-coalgebras is a morphism of coalgebras $f: C \rightarrow D$, satisfying the additional condition that for all $c \in C, f\left(c^{\circ}\right)=f(c)^{\diamond}$.

(3) A subcoalgebra $D$ of a o-coalgebra satisfying $D^{\circ}=D$ is called a o-subcoalgebra.

In explicit terms and with respect to the comultiplication, the compatibility condition for $\circ$ means that for all $c \in C$ if $\Delta(c)=\sum c_{1} \otimes c_{2}$, then $\Delta\left(c^{\circ}\right)=\sum c_{2}^{\circ} \otimes c_{1}^{\circ}$.

Observation 3.2. It follows immediately from the definition above that $\varepsilon\left(c^{\circ}\right)=\overline{\varepsilon(c)}$ for all $c \in C$. Hence viewing $\left(C_{c}, \Delta_{c}, \overline{\varepsilon_{c}}\right)$ as a coalgebra, the o-structure is a morphism of coalgebras $\circ:(C, \Delta, \varepsilon) \rightarrow\left(C_{c}, \Delta_{c}, \overline{\varepsilon_{c}}\right)^{\text {cop }}$.

Example 3.3. If $V$ is an arbitrary finite dimensional complex vector space with basis $\mathcal{B}=\left\{e_{1}, \cdots, e_{n}\right\}$, then the coalgebra $c(V)=V^{*} \otimes V$ can be equipped with a $\circ$-structure defined on the basis $\mathcal{B}$ as $\left(e^{j} \otimes e_{i}\right)^{\circ}=e^{i} \otimes e_{j}$.

The o-structure on the coalgebra $C$ endows the category of right $C$ comodules with an additional "duality functor" as presented in Definition 3.6. The reader should notice that we are not talking about duality in a technical sense.

First we recall the following duality constructions that make sense in the context of general coalgebras.

Definition 3.4. Let $C$ be an arbitrary coalgebra and $(V, \chi)$ a right finite dimensional $C$-comodule. The map $\chi^{r}: V^{*} \rightarrow C \otimes V^{*}$ defined by the formula: $\chi^{r}(f)=\sum f_{-1} \otimes f_{0}$ if and only if $\sum f_{-1} f_{0}(v)=\sum f\left(v_{0}\right) v_{1}$, is a left $C$-comodule structure on $V^{*}$. The pair $\left(V^{*}, \chi^{r}\right)$ is frequently abbreviated as $V^{r}$ and called the right adjoint of $V$. In this manner we have defined a contravariant functor $(-)^{r}: \mathcal{M}_{f}^{C} \rightarrow{ }^{C} \mathcal{M}_{f}$. Similarly we can define $(-)^{\ell}:{ }^{C} \mathcal{M}_{f} \rightarrow \mathcal{M}_{f}^{C}$ and $V^{\ell}$ will be called the left adjoint of $V$.

Definition 3.5. If $C$ is a o-coalgebra and $(W, \chi)$ a left $C$-comodule, then the map $\chi^{\circ}$ defined as $\chi^{\circ}=\operatorname{sw}(\circ \otimes \mathrm{id}) \chi_{c}: W_{c} \rightarrow W_{c} \otimes C$ is a right $C$-comodule structure on $W_{c}$, where sw stands for the usual switching map. This correspondence can be extended to define a covariant functor ${ }^{C} \mathcal{M} \rightarrow \mathcal{M}^{C}$.

Definition 3.6. Let $C$ be a o-coalgebra. If $(V, \chi) \in \mathcal{M}_{f}^{C}$, then the map $\chi^{r \circ}=\left(\chi^{r}\right)^{\circ}: V_{c}^{*} \rightarrow V_{c}^{*} \otimes C$ is a right $C$-comodule structure on $V_{c}^{*}$. In 
explicit terms, if $\chi(v)=\sum v_{0} \otimes v_{1}$ and $\chi^{r \circ}(f)=\sum f_{0} \otimes f_{1}$ then

$$
\sum \overline{f_{0}(v)} f_{1}=\sum \overline{f\left(v_{0}\right)} v_{1}^{\circ} \text {. }
$$

We call $\mathcal{D}$ the contravariant functor $\mathcal{M}_{f}^{C} \rightarrow \mathcal{M}_{f}^{C}$ defined on objects as above, i.e. $\mathcal{D}(V, \chi)=\left(V_{c}^{*}, \chi^{r \circ}\right)$. If $\mathcal{B}=\left\{e_{1}, \cdots, e_{n}\right\}$ is a basis of $V$ and $\mathcal{B}^{*}=\left\{e^{1}, \cdots, e^{n}\right\}$ is its dual basis in $V^{*}$, then the matrix coefficients of $\chi$ and $\chi^{r o}$ are related by

$$
\chi\left(e_{i}\right)=\sum_{j} e_{j} \otimes t_{j i}, \quad \chi^{r \circ}\left(e^{i}\right)=\sum_{j} e^{j} \otimes t_{i j}^{\circ}
$$

Observation 3.7. In the situation above, it is clear that for a finite dimensional $C$-comodule $V$, we have that $\operatorname{Coeff}(\mathcal{D}(V))=\operatorname{Coeff}(V)^{\circ}$.

Next lemma follows immediately from the fact that $\circ$ is an involution.

Lemma 3.8. If $C$ is a o-coalgebra, then $\mathcal{D}^{2}=(-)^{\ell r}: \mathcal{M}_{f}^{C} \rightarrow \mathcal{M}_{f}^{C}$.

In order to look at the adequate concept of representation for a ocoalgebra we need the following definition.

Definition 3.9. (1) Let $C$ be a o-coalgebra and $V \in \mathcal{M}^{C}$. A hermitian form $\beta: V \otimes V_{c} \rightarrow \mathbb{C}$ is unitary or invariant if $\sum \beta\left(u_{0}, v\right) u_{1}=$ $\sum \beta\left(u, v_{0}\right) v_{1}^{\circ}$, for all $u, v \in V$. If moreover, $\beta$ is an inner product, then it is called a unitary structure on $V$.

(2) A pair $(V,\langle\rangle$,$) consisting of a C$-comodule $V$ and a unitary structure on $V$ is called a unitary $C$-comodule. Moreover, if $(V,\langle\rangle$, and $(W,[]$,$) are unitary comodules, a morphism of comodules$ $f: V \rightarrow W$ is said to be unitary if it is an isometry with respect to $\langle$,$\rangle and [$,$] . Sometimes a unitary structure is called a$ unitary inner product.

Example 3.10. Let $V$ be a finite dimensional vector space and consider the o-coalgebra $c(V)$ of Example 3.3. Then, the inner product of $V$ that makes the given basis $\mathcal{B}$ orthonormal, is a unitary form in the $c(V)$-comodule $V$.

Lemma 3.11. Let $C$ be a o-coalgebra, $(V, \chi) \in \mathcal{M}_{f}^{C}$ and let $\beta: V \otimes V_{c} \rightarrow \mathbb{C}$ be a hermitian form on $V$.

Then, the following assertions are equivalent:

(1) The form $\beta$ is unitary.

(2) The map $\psi_{\beta}: V=(V, \chi) \rightarrow \mathcal{D}(V)=\left(V_{c}^{*}, \chi^{r \circ}\right)$-see Observation 2.4is a morphism in $\mathcal{M}_{f}^{C}$.

Corollary 3.12. If $\beta$ is a unitary structure on the $C$-comodule $V$, then $\operatorname{Coeff}(V) \subset C$ is a o-subcoalgebra that is simple if $V$ is irreducible. 
Proof. In this situation $\psi_{\beta}$ is an isomorphism, then the result follows from Observations 2.3 and 3.7 from which we deduce that $\operatorname{Coeff}(V)=\operatorname{Coeff}\left(V_{c}^{*}\right)$ $=\operatorname{Coeff}(V)^{\circ}$.

Corollary 3.13. Assume that $C$ is a o-coalgebra, that $V$ is a $C$-comodule and that $\beta: V \otimes V_{c} \rightarrow \mathbb{C}$ is a unitary structure on $V$. If $V_{\lambda}$ and $V_{\mu}$ are irreducible non isomorphic subcomodules of $V$, then $V_{\lambda}$ and $V_{\mu}$ are $\beta$ orthogonal.

Proof. Consider $C_{\lambda}=\operatorname{Coeff}\left(V_{\lambda}\right) \subset C$ and $C_{\mu}=\operatorname{Coeff}\left(V_{\mu}\right) \subset C$, the simple subcoalgebras of coefficients associated to $V_{\lambda}$ and $V_{\mu}$ respectively. As $V_{\lambda}$ and $V_{\mu}$ are not isomorphic, then $C_{\lambda} \cap C_{\mu}=\{0\}$-see Observation 2.3-. In this situation, for $u \in V_{\lambda}$ and $v \in V_{\mu}$, if we apply $\varepsilon$ to the equality $\sum \beta\left(u_{0}, v\right) u_{1}=\sum \beta\left(u, v_{0}\right) v_{1}^{\circ} \in C_{\lambda} \cap C_{\mu}^{\circ}=C_{\lambda} \cap C_{\mu}=\{0\}$, we deduce that $\beta(u, v)=0$.

In the presence of a unitary structure the comments appearing in Observation 2.2 can be refined.

Lemma 3.14. Assume that $C$ is an arbitrary $\circ-$ coalgebra and that $(V, \chi)$ is a finite dimensional right $C$-comodule. Choose a basis $\mathcal{B}=\left\{e_{1}, \cdots, e_{n}\right\}$ and define the matrix $T=\left(t_{i j}\right)_{1 \leq i, j \leq n}$ by the formula $\chi\left(e_{i}\right)=\sum_{j} e_{j} \otimes t_{j i}$. Then $T^{\circ}=T^{t}$ if and only if the inner product that makes the basis $\mathcal{B}$ orthonormal, is a unitary structure.

Proof. First we observe that if the product is unitary, then the condition $T^{\circ}=T^{t}$ is satisfied. The unitary condition $\sum\left\langle u_{0}, v\right\rangle u_{1}=\sum\left\langle u, v_{0}\right\rangle v_{1}^{\circ}$ applied to a pair of basis vectors $u=e_{k}$ and $v=e_{j}$ yields directly the equality $t_{k i}^{\circ}=t_{i k}$, i.e., $T^{\circ}=T^{t}$. Conversely, if this condition is satisfied, the above reasoning can be reversed and one proves that the inner product is unitary.

Observation 3.15. If $C$ is a o-coalgebra and $(V,\langle\rangle$,$) is a unitary C$ comodule, it is clear from the definitions that if $W \subset V$ is an arbitrary $C$-subcomodule, then the orthogonal complement $W^{\perp}$ of $W$ is also a $C$ subcomodule of $V$ (see also [1, Corollary 5.16] for details). Hence a unitary $C$-comodule is completely reducible.

Definition 3.16. If $C$ is a o-coalgebra, we say that $C$ is compact if every right $C$-comodule admits a unitary structure as introduced in Definition 3.9 .

Observation 3.17. (1) It follows from Observation 3.15 that a compact coalgebra is cosemisimple. 
(2) If $C$ is compact, and we consider it as a right $C$-comodule, then it can be endowed with a unitary inner product that will satisfy the following condition: for all $c, d \in C, \sum\left\langle c_{1}, d\right\rangle c_{2}=\sum\left\langle c, d_{1}\right\rangle d_{2}^{\circ}$.

(3) In the situation above if $1 \in \mathbb{C}$ is a group like element with the property that $1^{\circ}=1$, we can define a map $\varphi: C \rightarrow \mathbb{C}$, as $\varphi(c)=$ $\langle c, 1\rangle \in \mathbb{C}$. It is clear that $\varphi$ is a right integral on $C$ with $\varphi(1)=$ 1. Indeed, $\sum \varphi\left(c_{1}\right) c_{2}=\sum\left\langle c_{1}, 1\right\rangle c_{2}=\langle c, 1\rangle 1^{\circ}=\varphi(c) 1$. Moreover $\varphi(1)=\langle 1,1\rangle>0$ because we are dealing with inner products.

\section{Compactness, cosemisimplicity and Fourier forms}

In the presence of a o-structure on $C$ the existence of inner products can be related with the theory of Fourier forms and some of the considerations of the preceeding section can be refined.

Definition 4.1. Assume that $C$ is a o-coalgebra, that $\omega$ is a Fourier form for $C$ and that $\star$ is a Fourier product.

(1) We say that $\omega$ is positive if and only if $\omega\left(c^{\circ}, c\right) \geq 0$ for all $c \in C$. If $\omega\left(c^{\circ}, c\right)>0$ whenever $c \neq 0$ we say that $\omega$ is positive definite.

(2) We say that $\omega$ is hermitian if for all $c, d \in C, \omega\left(c^{\circ}, d^{\circ}\right)=\overline{\omega(d, c)}$.

(3) We say that $\star$ is positive if and only if $\varepsilon\left(c^{\circ} \star c\right) \geq 0$ for all $c \in C$. If $\varepsilon\left(c^{\circ} \star c\right)>0$ whenever $c \neq 0$ we say that $\star$ is positive definite.

(4) We say that $\star$ is hermitian if for all $c, d \in C, c^{\circ} \star d^{\circ}=(d \star c)^{\circ}$.

It follows directly from the above definition that the Fourier form $\omega$ is positive (positive definite, hermitian) if and only if the corresponding Fourier product $\star_{\omega}$ is positive (respectively positive definite, hermitian).

Observation 4.2. (1) In the case that $C$ has a o-structure and also a Fourier form $\omega$, the map $\langle,\rangle_{\omega}: C \otimes C \rightarrow \mathbb{C}$ defined for all $c, d \in C$ as $\langle c, d\rangle_{\omega}=\omega\left(d^{\circ}, c\right)$ is a unitary sesquilinear form in $C$.

(2) Hence, in the situation of a o-coalgebra $C$, there is a bijective correspondence between Fourier forms, Fourier products and unitary sesquilinear forms on $C$ and this correspondence is given by the following rules: $\omega \mapsto \star_{\omega}, \star \mapsto\langle,\rangle_{\star}$ and $\langle,\rangle \mapsto \omega_{\langle,\rangle}$where for all $c, d \in C, c \star_{\omega} d=\sum \omega\left(c, d_{1}\right) d_{2}=\sum c_{1} \omega\left(c_{2}, d\right),\langle c, d\rangle_{\star}=\varepsilon\left(d^{\circ} \star c\right)$ and $\omega_{\langle,\rangle}(c, d)=\left\langle d, c^{\circ}\right\rangle$.

(3) The direct expression of the Fourier product in terms of the corresponding unitary sesquilinear form is the following: for all $c, d \in C$ : $c \star d=\sum\left\langle d_{1}, c^{\circ}\right\rangle d_{2}=\sum c_{1}\left\langle d, c_{2}^{\circ}\right\rangle$.

(4) The Fourier form $\omega$ is symmetric if and only if the associated sesquilinear form satisfies the following condition: $\left\langle c^{\circ}, d^{\circ}\right\rangle=\langle d, c\rangle$. In case that the sesquilinear form is hermitian, the symmetry of $\omega$ is equivalent to the condition $\left\langle c^{\circ}, d^{\circ}\right\rangle=\overline{\langle c, d\rangle}$. 
(5) The correspondence considered above between Fourier forms and unitary sesquilinear forms in $C$, preserves positivity and hermitianity. Hence, in $C$ there is a bijective correspondence between positive definite hermitian Fourier forms and unitary inner products.

The theorem below refines for the case of compact coalgebras, a well known result on the structure of cosemisimple coalgebras.

Theorem 4.3. Let $C$ be a o-coalgebra. The following properties are equivalent.

(1) The coalgebra $C$ is compact.

(2) The coalgebra $C$ viewed as a right $C$-comodule admits a unitary inner product.

(3) The coalgebra $C$ admits a Fourier form that is positive definite and hermitian.

(4) The coalgebra $C$ admits a Fourier product that is positive definite and hermitian.

(5) The coalgebra $C$ can be decomposed as $C=\bigoplus_{\rho \in \widehat{C}} C_{\rho}$-for some set of subindexes that we call $\widehat{C}_{-}^{-}$, where each $C_{\rho} \subset C$ is a simple o-subcoalgebra. Moreover, each $C_{\rho}$ admits a basis of elements $\left\{t_{i j}^{\rho}\right.$ : $\left.1 \leq i, j \leq n_{\rho}\right\}$ such that for all $1 \leq i, j \leq n_{\rho}, \Delta\left(t_{i j}^{\rho}\right)=\sum_{k} t_{i k}^{\rho} \otimes t_{k j}^{\rho}$ and $t_{i j}^{\rho \circ}=t_{j i}^{\rho}$.

Proof. It is clear that condition (1) implies condition (2) and the proof that (2), (3) and (4) are equivalent is the content of Observation 4.2.

To prove that (2) implies (5) we proceed as follows. First recall that $C$ is cosemisimple (see Observation 3.17) and write $C=\bigoplus_{\rho \in \widehat{C}} C_{\rho}$ with $C_{\rho}$ a simple subcoalgebra of $C$. It follows from Corollary 3.12 that each $C_{\rho}$ is a o-coalgebra and from Observation 2.3 that the family of all $C_{\rho}$ for $\rho \in \widehat{C}$ consists of all simple subcoalgebras of $C$.

Hence, we have finished the proof of first part of (5). Now, call $V_{\rho}$ an irreducible right coideal with the property that $\operatorname{Coeff}\left(V_{\rho}\right)=C_{\rho}$ and take a basis $\mathcal{B}_{\rho}=\left\{e_{1}^{\rho}, \cdots, e_{n_{\rho}}^{\rho}\right\}$ of $V_{\rho}$ that is orthogonal with respect to the given unitary inner product in $C$, and consider the basis $\left\{t_{i j}^{\rho}: 1 \leq i, j \leq n_{\rho}\right\}$ of $C_{\rho}$ defined by the formulæ: $\Delta\left(e_{i}^{\rho}\right)=\sum_{j} e_{j}^{\rho} \otimes t_{j i}^{\rho}$-see Observation 2.2-. In accordance with Lemma 3.14, the elements $\left\{t_{i j}^{\rho}: 1 \leq i, j \leq n_{\rho}\right\}$ satisfy the required properties: $\Delta\left(t_{i j}^{\rho}\right)=\sum_{k} t_{i k}^{\rho} \otimes t_{k j}^{\rho}$ and $t_{i j}^{\rho \circ}=t_{j i}^{\rho}$.

Now, assuming condition (5) we prove condition (1) as follows. It is well known, see for example [1, Theorem 4.1], that being $C$ the direct sum 
of simple subcoalgebras, the category of right $C$-comodules is semisimple. We want to prove that any $C$-comodule is unitary and from the above it follows that it is enough to prove that irreducible $C$-comodules are unitary. Indeed, in order to construct a unitary product in an arbitrary $C$-comodule, we define the product in its irreducible components and then extend it by forcing irreducible non isomorphic components to be orthogonal.

If $V$ is an irreducible $C$-comodule, then $\operatorname{Coeff}(V)$ is of the form $C_{\rho}$ for some $\rho \in \widehat{C}$ and then $V \subset C_{\rho}$ for some $\rho$. We define the inner product $\langle$,$\rangle in C_{\rho}$ setting $\left\langle t_{i k}^{\rho}, t_{j \ell}^{\rho}\right\rangle=\delta_{i j} \delta_{k \ell}$ and then by restriction we define it in $V$. In particular it is clear that the inner product thus defined is unitary.

Notice that condition (4) above, guarantees that each $C_{\rho}$ as a o-coalgebra is isomorphic to $\left(M_{n}(\mathbb{C}),(-)^{*}\right)$, that is the usual matrix coalgebra with the o-operation given by the adjoint operator.

Observation 4.4. It is important to remark that the inner product constructed along the proof of part (5) of the above theorem, satisfies the following additional condition:

$$
\left\langle c^{\circ}, d^{\circ}\right\rangle=\overline{\langle c, d\rangle}, \quad \forall c, d \in C .
$$

Equivalently -see Observation 4.2, (4)- the corresponding associated Fourier form is symmetric.

\section{The case of Hopf algebras}

From now on we assume that our basic ${ }^{\circ-}$ coalgebra $H$ has the additional structure of a Hopf algebra with a product that is compatible with the ostructure.

Definition 5.1. Assume that $H$ is a Hopf algebra and that $\circ$ is a $\circ-$ structure on $(H, \Delta, \varepsilon)$. We say that the pair $(H, \circ)$ is a $\circ-H o p f$ algebra if $(x y)^{\circ}=x^{\circ} y^{\circ}$, for all $x, y \in H$.

In this situation, -see [15]- the following properties are satisfied: $1^{0}=1$, and $S \circ S \circ=$ id. In particular, it is clear that $\circ: H \rightarrow H_{c}$ is an algebra automorphism and that the antipode of $H$ is an invertible linear map with inverse $S^{-1}=\circ S \circ$.

Next, we compare this definition with the equivalent concept of $\star-$ Hopf algebra.

Recall that a $\star$-Hopf algebra, is a Hopf algebra $H$ defined over $\mathbb{C}$ and equipped with a conjugate linear involution $\star: H \rightarrow H$, such that $H$ : it is a $\star$-algebra, and the maps $\Delta: H \rightarrow H \otimes H$ and $\varepsilon$ are $\star$-homomorphisms. 
The concept of $\star-$ Hopf algebra and of o-Hopf algebra are equivalent as for a given $\star$-structure the map $S \star$ is obviously a $\circ$-structure on $H$.

Next we collect for future reference some of the basic properties of Fourier forms, products and integrals in the case of a Hopf algebra.

Observation 5.2. The considerations that follow are consequence of Observations 4.2. If $H$ is a ${ }^{-}$-Hopf algebra and $\varphi$ is a right integral on $H$, then

$$
\langle x, y\rangle_{\varphi}=\varphi\left(S\left(y^{\circ}\right) x\right), \quad \forall x, y \in H
$$

defines a unitary sesquilinear form $\langle,\rangle_{\varphi}: H \otimes H_{c} \rightarrow \mathbb{C}$. Moreover,

(1) $\langle,\rangle_{\varphi}$ is hermitian if and only if $\varphi\left(x^{\circ}\right)=\overline{\varphi(S(x))}, \forall x \in H$,

(2) $\langle,\rangle_{\varphi}$ is positive definite if and only if $\varphi\left(S\left(x^{\circ}\right) x\right)>0, \forall x \neq 0 \in H$.

We prove one of the implications of part (1), the rest of the proof is direct.

$$
\begin{gathered}
\varphi\left(S\left(x^{\circ}\right) y\right)=\varphi\left(\left(S\left(x^{\circ}\right)^{\circ} y^{\circ}\right)^{\circ}\right)= \\
\varphi\left(\left(S^{-1}(x) y^{\circ}\right)^{\circ}\right)=\overline{\varphi\left(S\left(S^{-1}(x) y^{\circ}\right)\right)}=\overline{\varphi\left(S\left(y^{\circ}\right) x\right)} .
\end{gathered}
$$

Observation 5.3. (1) In the situation of a Hopf algebra $H$, if $(W, \chi) \in$ ${ }^{H} \mathcal{M}$ and $\chi^{S}=\operatorname{sw}(S \otimes$ id $) \chi: W \rightarrow W \otimes H$, then $\left(W, \chi^{S}\right) \in \mathcal{M}^{H}$. We can consider also the standard duality functor $(V, \chi) \mapsto\left(V^{*}, \chi^{r S}\right): \mathcal{M}_{f}^{H} \rightarrow \mathcal{M}_{f}^{H}$, that defines a duality in the category $\mathcal{M}_{f}^{H}$. In explicit terms $\chi^{r S}(f)=\sum f_{0} \otimes f_{1}$, if and only if $\sum f_{0}(v) f_{1}=\sum f\left(v_{0}\right) S\left(v_{1}\right)$ where $\chi(v)=\sum v_{0} \otimes v_{1}$.

(2) It follows directly from the above formula that the cannonical map $j_{V}:\left(V,\left(\mathrm{id} \otimes S^{2}\right) \chi\right) \rightarrow\left(V^{* *}, \chi^{r S r S}\right)$ is a morphism of $H$-comodules.

(3) In the situation above, it is clear that Coeff $\left(V^{*}, \chi^{r S}\right)=$ $S(\operatorname{Coeff}(V, \chi))$. It is also interesting at this point to recall that $\operatorname{Coeff}\left(V_{c}^{*}, \chi^{r \circ}\right)=\operatorname{Coeff}(V, \chi)^{\circ}$-see Observation 3.7-.

Lemma 5.4. Assume that $H$ is a $\circ$-Hopf algebra and let $(V, \chi)$ be a finite dimensional right $H$-comodule that admits an unitary inner product $\gamma$ : $V^{*} \otimes V_{c}^{*} \rightarrow \mathbb{C}$. Then the map $\psi_{\gamma_{c}}:\left(V_{c}^{*}, \chi^{r \circ}\right) \rightarrow\left(V^{* *}, \chi^{r S r S}\right)$ is a morphism of $H$-comodules.

Proof. In the following proof we call $\chi^{r o}(f)=\sum f_{(0)} \otimes f_{(1)}$ and $\chi^{r S}(f)=$ $\sum f_{0} \otimes f_{1}$. 
We want to prove that the following diagram is commutative

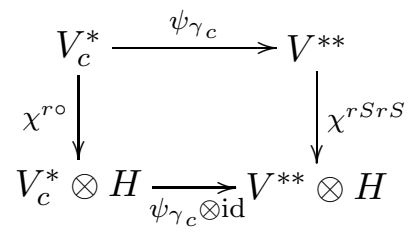

As $j_{V}:\left(V,\left(\mathrm{id} \otimes S^{2}\right) \chi\right) \rightarrow\left(V^{* *}, \chi^{r S r S}\right)$ is a morphism -see Observation 5.3 - the commutativity of $(2)$ is equivalent to the commutativity of:

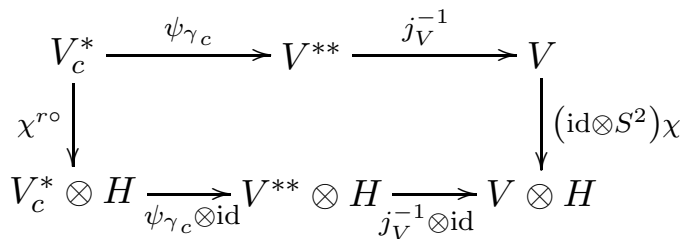

Let be $f \in V_{c}^{*}$ and call $j_{V}^{-1}\left(\psi_{\gamma_{c}}(f)\right)=v$. Then

$$
\gamma(h, f)=h(v), \quad \forall h \in V^{*} .
$$

We have to show that

$$
\sum \psi_{\gamma_{c}}\left(f_{(0)}\right) \otimes f_{(1)}=\sum j_{V}\left(v_{0}\right) \otimes S^{2}\left(v_{1}\right)
$$

After evaluation at an arbitrary $g \in V^{*}$ and using (3) and Observation 5.3, (1); the above equation becomes:

$$
\sum \gamma\left(g, f_{(0)}\right) f_{(1)}=S\left(\sum \gamma\left(g_{0}, f\right) g_{1}\right) .
$$

Let us consider a fixed $g \in V^{*}$ and represent it by an unique $w \in V$ such that $\overline{\gamma(g,-)}=j_{V}(w)$, i.e.:

$$
\gamma(g, h)=\overline{h(w)}, \quad \forall h \in V^{*} .
$$

Hence using the characterization of $\chi^{r \circ}$ given in Definition 3.6, equation (4) becomes:

$$
\sum \overline{f\left(w_{0}\right)} w_{1}^{\circ}=S\left(\sum \gamma\left(g_{0}, f\right) g_{1}\right) \in H
$$

Next observe that the condition of unitarity of $\gamma, \sum \gamma\left(g, f_{0}\right) f_{1}^{\circ}=$ $\sum \gamma\left(g_{0}, f\right) g_{1}$, expressed in terms of $w$ instead of $g$ becomes:

$\sum \overline{f_{0}(w)} f_{1}^{\circ}=\sum \gamma\left(g_{0}, f\right) g_{1}$, and after applying the antipode $S$ we have: $S \circ\left(\sum f_{0}(w) f_{1}\right)=S\left(\sum \gamma\left(g_{0}, f\right) g_{1}\right)$. Going back to equation (4) we have: 


$$
\begin{aligned}
S\left(\sum \gamma\left(g_{0}, f\right) g_{1}\right) & =S \circ\left(\sum f_{0}(w) f_{1}\right)=\circ S^{-1}\left(\sum f_{0}(w) f_{1}\right) \\
& =\circ S^{-1}\left(\sum f\left(w_{0}\right) S\left(w_{1}\right)\right)=\sum \overline{f\left(w_{0}\right)} w_{1}^{\circ} .
\end{aligned}
$$

The following theorem will be of crucial importance later in the treatment of compact involutions. Notice that this result is related to [4, Theorem 1.7] and to [14, Theorem 3.3].

Theorem 5.5. Assume that $H$ is a o-Hopf algebra and let $(V, \chi)$ be a finite dimensional right $H$-comodule with the property that $V$ as well as $V^{*}$ admit unitary inner products. Then there is a positive definite isomorphism of comodules between $(V, \chi)$ and $\left(V,\left(\mathrm{id} \otimes S^{2}\right) \chi\right)$.

Proof. We denote the inner products as $\beta: V \otimes V_{c} \rightarrow \mathbb{C}$ and $\gamma: V^{*} \otimes V_{c}^{*} \rightarrow$ $\mathbb{C}$ and call $\psi_{\beta}: V \rightarrow V_{c}^{*}$ and $\psi_{\gamma}: V^{*} \rightarrow V_{c}^{* *}$ the corresponding linear isomorphisms.

The Lemma 3.11 and Observation 5.3 guarantee that the maps $\psi_{\beta}$ : $(V, \chi) \rightarrow\left(V_{c}^{*}, \chi^{r \circ}\right)$ and $j_{V}:\left(V,\left(\mathrm{id} \otimes S^{2}\right) \chi\right) \rightarrow\left(V^{* *}, \chi^{r S r S}\right)$ are morphisms of $H$-comodules. Moreover, in accordance with Observation 2.4, the map $\phi$ defined by the diagram below is bijective and positive definite with respect to the inner product $\beta$ of $V$

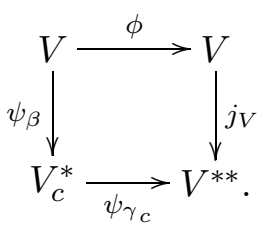

We prove that the map $\phi:(V, \chi) \rightarrow\left(V,\left(\mathrm{id} \otimes S^{2}\right) \chi\right)$ is a morphism of $H$-comodules. 
Consider the diagram that follows:

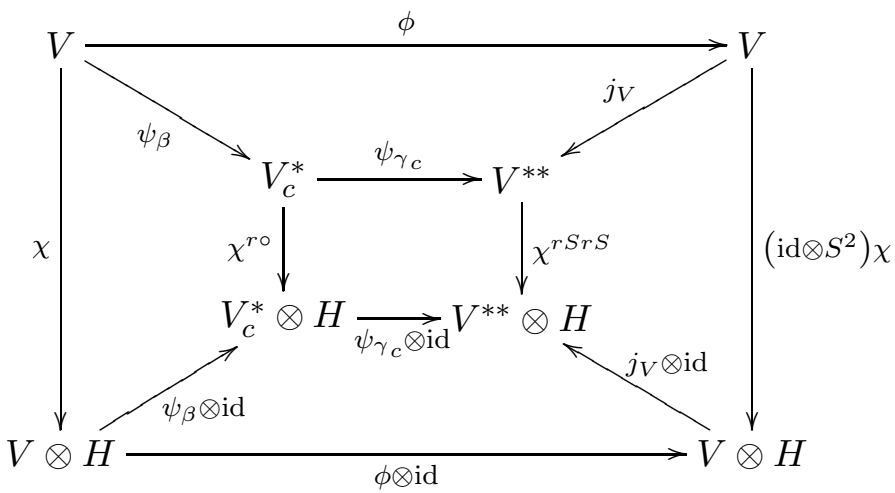

Of the four trapezoids that appear in the diagram, the upper and lower are commutative by $(7)$; the left and right because $\psi_{\beta}:(V, \chi) \rightarrow\left(V_{c}^{*}, \chi^{r \circ}\right)$ and $j_{V}:\left(V,\left(\mathrm{id} \otimes S^{2}\right) \chi\right) \rightarrow\left(V^{* *}, \chi^{r S r S}\right)$ are morphisms of $H$-comodules, respectively. As the square in the center is commutative by the above Lemma 5.4, we conclude that the exterior rectangle is commutative, i.e. that $\phi:(V, \chi) \rightarrow\left(V,\left(\mathrm{id} \otimes S^{2}\right) \chi\right)$ is an $H$-comodule map.

\section{Compact Quantum Groups}

In this section we review some of the basic definitions related to the concept of compact quantum group. See [27] for the original definition and also the papers cited in the Introduction for the early development of the subject.

Definition 6.1. [1, Definition 7.1, Proposition 7.5] Assume that $(H, \Delta, \varepsilon, \mu, 1, S, \circ)$ is a o-Hopf algebra. We say that $H$ is a compact quantum group if $(H, \Delta, \varepsilon, \circ)$ is a compact o-coalgebra.

In other words, a compact quantum group is a o-Hopf algebra with the additional property that all the $H$-comodules can be endowed with a unitary innner product.

Recall that in the situation of the equivalent set up of $\star$-Hopf algebras -see for example [13]-, an inner product in an $H$-comodule $V$ is called unitary if it satisfies the following additional condition: for all $v, w \in V$ we have that $\sum\left\langle v_{0}, w_{0}\right\rangle w_{1}^{\star} v_{1}=\langle v, w\rangle$. If we define $\circ=\star S$ this unitary condition is equivalent to the one appearing in Definition 3.9. Now, the definition of compact quantum group in terms of a $\star$ operation is that of a *-Hopf algebra with the property that all the $H$-comodules admit a unitary inner product. Hence the definition of compact quantum group apperaring 
for example in [3], [4] or [13] in terms of a $\star$-structure on $H$, is equivalent to the one we presented above.

As we mentioned before, the above Definition 6.1 of compact quantum group -or the equivalent concept in terms of a $\star$-operation- is the algebraic counterpart of the original definition by Woronowicz, that is obtained from the one above by a process of "completion". We refer the reader to [3, Proposition 1.4], [10, Chapter 2, Section 4] or [8] for the precise description of this relationship.

We recall some results concerning cosemisimple Hopf algebras and then look in the case of o-Hopf algebras at the behavior of the integral in relation to the o-operator.

Observation 6.2. (1) Suppose that $H$ is a cosemisimple Hopf algebra. We can consider $\mathbb{C}$ as a right -or left- $H$-comodule with the trivial structure given by the unit. In Observation 2.3 we noticed that we can write $H=\bigoplus_{\rho \in \widehat{H}} H_{\rho}$ where $H_{\rho}$ is the family of all simple subcoalgebras of $H$. In this situation we call $\rho_{0}$ the element in $\widehat{H}$, with the property that $H_{\rho_{0}}=\mathbb{C}$. Let $\varphi: H \rightarrow \mathbb{C}$ be the projection from $H$ onto the component $H_{\rho_{0}}$. It is clear that $\varphi$ is a morphism of right and left comodules and this implies that it is a right and left normal integral on $H$.

(2) In particular, the antipode $S$ permutes the simple subcoalgebras $H_{\rho}$ for $\rho \in \widehat{H}$ and leaves $H_{\rho_{0}}=\mathbb{C}$ fixed.

(3) In this situation if $\varphi: H \rightarrow \mathbb{C}$ is the normal -right and left-integral on $H$, we can consider in $H$ the Fourier product $\star_{\varphi}$ and the normal Fourier form $\omega_{\varphi}$ induced by $\varphi$-see Observation $5.2-$.

$$
\begin{gathered}
x \star_{\varphi} y=\sum \varphi\left(S(x) y_{1}\right) y_{2}=\sum x_{1} \varphi\left(S\left(x_{2}\right) y\right), \quad \forall x, y \in H . \\
\omega_{\varphi}(x, y)=\varphi(S(x) y) .
\end{gathered}
$$

The Fourier form considered above is left and right non degenerate and satisfies:

$$
\omega_{\varphi}(x, y)=\omega_{\varphi}\left(y, S^{2}(x)\right), \quad \forall x, y \in H_{\rho} .
$$

See for example [20], for the non degeneracy of the form.

(4) Moreover, in the case of a o-Hopf algebra, the map $\circ: H \rightarrow H$ leaves invariant all the $H_{\rho}$, including $H_{\rho_{0}}$-see Lemma 3.11-. Hence it follows that $\varphi \circ=\bar{\varphi}$, i.e., $\varphi\left(x^{\circ}\right)=\overline{\varphi(x)}$ for all $x \in H$.

(5) The bilinear map $\langle,\rangle_{\varphi},\langle x, y\rangle_{\varphi}=\varphi\left(S\left(y^{\circ}\right) x\right)$, for all $x, y \in H$, is hermitian, non degenerate and unitary. The hermitianity is equivalent to the property that $\varphi\left(x^{\circ}\right)=\overline{\varphi(S(x))}$-see Observation 5.2that is a consequence of the properties (1) and (4) above. 
Observation 6.3. (1) It follows from Theorem 5.5 that for an arbitrary simple coalgebra $C \subset H$ of the compact quantum group $H$, we have that $S^{2}(C)=C$. Indeed, in accordance with the mentioned theorem, if $V$ is a finite dimensional $H$-comodule, there is an isomorphism of $H$-comodules $\varphi:(V, \chi) \rightarrow\left(V\right.$, (id $\left.\left.\otimes S^{2}\right) \chi\right)$. Hence, Coeff $(V)=S^{2}(\operatorname{Coeff}(V))$ and taking $V$ an irreducible comodule that has $C$ as coalgebra of coefficients, we deduce that $C=S^{2}(C)$. Notice that this result is valid for any simple subcoalgebra $C$ of a Hopf algebra - not necessarily with a o-structure- and was proved by Larson in [14][Thm. 3.3].

(2) For future reference we write down the following formula, valid in our context for all $x, y \in H$

$$
\sum x_{1}\left\langle x_{2}, y\right\rangle_{\varphi}=\sum S^{2}\left(y_{1}^{\circ}\right)\left\langle x, y_{2}\right\rangle_{\varphi}
$$

Explicitly, we need to show that:

$\sum x_{1} \varphi\left(S\left(y^{\circ}\right) x_{2}\right)=\sum S^{2}\left(y_{1}^{\circ}\right) \varphi\left(S\left(y_{2}^{\circ}\right) x\right)$, that by a change of variables becomes: $\sum x_{1} \varphi\left(y x_{2}\right)=\sum S\left(y_{1}\right) \varphi\left(y_{2} x\right)$. This last equality can be proved directly using the fact that $\varphi$ is an integral -see [9]-.

Let $H$ be a compact quantum group, next we prove that the non degenerate unitary form associated to the integral -that is denoted as $\langle,\rangle_{\varphi^{-}}$ and given explicitly as $\langle x, y\rangle=\varphi\left(S\left(y^{\circ}\right) x\right)$ is in fact an inner product. This is the content of Theorem 6.7 .

In order to prove this positivity result we will use the unitary inner product whose existence was established along the proof of Theorem 4.3. This product will be denoted as $\langle$,$\rangle and it satisfies the equation \left\langle x^{\circ}, y^{\circ}\right\rangle=$ $\overline{\langle x, y\rangle}$-see Observation 4.4, (1)-.

As the Fourier forms associated to $\langle,\rangle_{\varphi}$ and $\langle$,$\rangle are unitary, the sub-$ coalgebras $H_{\rho}$ are orthogonal with respect to both. The mentioned positivity result in $H$ will be deduced of the corresponding result for each $H_{\rho}$. Explicitly the Fourier products $\star$ and $\star_{\varphi}$ associated respectively with $\langle$, and $\langle,\rangle_{\varphi}$ are given as:

$$
\begin{aligned}
x \star y & =\sum\left\langle y_{1}, x^{\circ}\right\rangle_{2}=\sum x_{1}\left\langle y, x_{2}^{\circ}\right\rangle, \\
x \star_{\varphi} y & =\sum\left\langle y_{1}, x^{\circ}\right\rangle_{\varphi} y_{2}=\sum x_{1}\left\langle y, x_{2}^{\circ}\right\rangle_{\varphi}=\sum \varphi\left(S(x) y_{1}\right) y_{2}= \\
& =\sum x_{1} \varphi\left(S\left(x_{2}\right) y\right) .
\end{aligned}
$$

Next Lemmas 6.4, 6.5 are preparatory for the proof ot the positivity result mentioned above.

Lemma 6.4. Let $H$ be a compact quantum group and let $H_{\rho}$ be one of its simple components as before. If $H_{\rho}=\bigoplus_{a} R_{a}$ is the decomposition 
of $H_{\rho}$ into irreducible right coideals (that are pairwise $\langle$,$\rangle -orthogonal in$ accordance with Corollary 3.13), then:

(1) $H_{\rho}=\bigoplus_{a}\left(R_{a}\right)^{\circ}$ is also a decomposition of $H_{\rho}$ into pairwise orthogonal subspaces.

(2) The element $s_{\rho} \in H_{\rho}$, neutral for the restriction of $\star$ to $H_{\rho}$, is o-stable, i.e., $s_{\rho}=s_{\rho}^{\circ}$.

(3) If we write $s_{\rho}=\sum_{a} s_{a}$, with $s_{a} \in R_{a}$ then, for all $x \in R_{a}, x=s_{a} \star x$ and if $b \neq a$, then $s_{b} \star x=0$. In particular $s_{a} \neq 0$, for all $a$.

Proof. (1) The properties of this decomposition can be easily deduced from the fact that $R_{a}$ is orthogonal to $R_{b}$ when $a \neq b$-see Corollary 3.13-(we are also using that for $x, y \in H_{\rho},\left\langle x^{\circ}, y^{\circ}\right\rangle=\overline{\langle x, y\rangle}$, c.f. Observation 4.4).

(2) The existence of $s_{\rho}$ follows from Observation 2.6. We have that: $s_{\rho}^{\circ}=s_{\rho}^{\circ} \star s_{\rho}=\left(s_{\rho}^{\circ} \star s_{\rho}\right)^{\circ}=\left(s_{\rho}^{\circ}\right)^{\circ}=s_{\rho}$-see Observation 4.2-.

(3) From the equality: $x=s_{\rho} \star x=\sum_{b}\left(s_{b} \star x\right)$ and the fact that $s_{b} \star x \in R_{b}$ for all $b$-see Observation 2.6, we deduce our result.

Lemma 6.5. Let $H$ be a compact quantum group, let $H_{\rho}$ be one of its simple components and let $H_{\rho}=\bigoplus_{a} R_{a}$ be the decomposition of the simple subcoalgebra $H_{\rho}$ into irreducible right coideals. Then, for each a we call $A_{a}: R_{a} \rightarrow R_{a}$ the linear map defined by the equality:

$$
A_{a}(x)=s_{a} \star_{\varphi} S^{2}(x), \quad \forall x \in R_{a} .
$$

Then:

(1) For all $x, y \in R_{a}$, we have

$$
\left\langle y^{\circ}, x^{\circ}\right\rangle_{\varphi}=\left\langle A_{a}(x), y\right\rangle .
$$

(2) The map $A_{a}$ is a positive operator with respect to the inner product $\langle$,$\rangle .$

Proof. (1) To prove the equality (9), we take $x, y \in R_{a}$ and deduce that:

$$
\begin{aligned}
\left\langle y^{\circ}, x^{\circ}\right\rangle_{\varphi} & =\varepsilon\left(x \star_{\varphi} y^{\circ}\right)=\varepsilon\left(y^{\circ} \star_{\varphi} S^{2}(x)\right)=\varepsilon\left(y^{\circ} \star_{\rho} \star_{\varphi} S^{2}(x)\right) \\
& =\left\langle s_{\rho} \star_{\varphi} S^{2}(x), y\right\rangle=\sum_{b}\left\langle s_{b} \star_{\varphi} S^{2}(x), y\right\rangle=\left\langle A_{a}(x), y\right\rangle .
\end{aligned}
$$

The second in the chain of equalities above comes from the fact that:

$$
\varepsilon\left(x \star_{\varphi} y^{\circ}\right)=\omega_{\varphi}\left(x, y^{\circ}\right)=\omega_{\varphi}\left(y^{\circ}, S^{2}(x)\right)=\varepsilon\left(y^{\circ} \star_{\varphi} S^{2}(x)\right),
$$

-see Observation 6.2 part (3)-. 
(2) The map $A_{a}:\left(R_{a},\left(\mathrm{id} \otimes S^{2}\right) \Delta\right) \rightarrow\left(R_{a}, \Delta\right)$ is an $H$-comodule map. Indeed,

$$
\begin{aligned}
\sum A_{a}\left(x_{1}\right) \otimes S^{2}\left(x_{2}\right) & =\sum s_{a} \star_{\varphi} S^{2}\left(x_{1}\right) \otimes S^{2}\left(x_{2}\right)= \\
& =\sum s_{a} \star_{\varphi} S^{2}(x)_{1} \otimes S^{2}(x)_{2}=(\text { see Def. } 2.5)= \\
& =\Delta\left(s_{a} \star_{\varphi} S^{2}(x)\right)=\Delta\left(A_{a}(x)\right) .
\end{aligned}
$$

From Theorem 5.5 and Schur's Lemma we deduce that there is a complex scalar $\gamma_{a}$ and a $\langle$,$\rangle -positive operator P_{a}$ on $R_{a}$ with the property that $A_{a}=\gamma_{a} P_{a}$. Let $B_{a}=\left\{e_{1}, e_{2}, \cdots, e_{n}\right\}$ be a $\langle$,$\rangle -orthonormal basis of R_{a}$. If we write $\Delta\left(s_{a}\right)=\sum_{i} e_{i} \otimes h_{i}$, then

$$
\sum_{i} e_{i}\left\langle e_{j}, h_{i}^{\circ}\right\rangle=s_{a} \star e_{j}=e_{j}
$$

so we deduce $\left\langle e_{j}, h_{i}^{\circ}\right\rangle=\delta_{i, j}$ and therefore $h_{i}=e_{i}^{\circ}$. Then, $\Delta\left(s_{a}\right)=\sum_{i} e_{i} \otimes e_{i}^{\circ}$ and -see Observation 2.6- $\sum_{i} e_{i} \star_{\varphi} e_{i}^{\circ}=s_{a}$. Applying $\varepsilon$ to this last equality we deduce that: $\sum_{i}\left\langle e_{i}^{\circ}, e_{i}^{\circ}\right\rangle_{\varphi}=\varepsilon\left(s_{a}\right)$.

The positivity of $\gamma_{a}$ follows from the following computation:

$$
\begin{gathered}
\gamma_{a} \sum_{i}\left\langle P_{a}\left(e_{i}\right), e_{i}\right\rangle=\sum_{i}\left\langle A_{a}\left(e_{i}\right), e_{i}\right\rangle=\sum_{i}\left\langle e_{i}^{\circ}, e_{i}^{\circ}\right\rangle_{\varphi}=\varepsilon\left(s_{a}\right)= \\
=\varepsilon\left(s_{\rho} \star s_{a}\right)=\left\langle s_{a}, s_{\rho}^{\circ}\right\rangle=\left\langle s_{a}, s_{\rho}\right\rangle=\left\langle s_{a}, s_{a}\right\rangle>0 .
\end{gathered}
$$

Hence $\gamma_{a}$ is a strictly positive real number and thus $A_{a}$ is a positive operator.

Observation 6.6. In particular for all $x \in R_{a}^{\circ}$,

$$
\langle x, x\rangle_{\varphi}=\left\langle A_{a}\left(x^{\circ}\right), x^{\circ}\right\rangle .
$$

Theorem 6.7. If $H$ is a compact quantum group and $\varphi: H \rightarrow \mathbb{C}$ is the associated normal integral, then the hermitian form $\langle,\rangle_{\varphi}$ is positive definite.

Proof. As we proved in Theorem 4.3 there is a decomposition

$H=\bigoplus_{\rho \in \widehat{H}} H_{\rho}$, where $H_{\rho}$ are non isomorphic simple subcoalgebras that are also o-stable and $\langle,\rangle_{\varphi}$-orthogonal. For each $\rho \in \widehat{H}$ we have a decomposition $H_{\rho}=\bigoplus_{a} R_{a}$, into irreducible right coideals that are all isomorphic -see Lemma 6.4-. Then, $H_{\rho}=\bigoplus_{a} R_{a}^{\circ}$ and we claim that the left coideals $R_{a}^{\circ}$ are $\langle,\rangle_{\varphi}$-orthogonal.

As $R_{a}$ and $R_{b}^{\circ}$ are respectively right and left coideals, from Observation 2.6 we deduce that $R_{a} \star R_{b}^{\circ} \subset R_{a} \cap R_{b}^{\circ}$. Conversely, if $x \in R_{a} \cap R_{b}^{\circ}$, then $x=s_{a} \star x \in R_{a} \star R_{b}^{\circ}$ and we deduce that $R_{a} \star R_{b}^{\circ}=R_{a} \cap R_{b}^{\circ}$. 
Applying $\varepsilon$ the the above equality we obtain:

$$
\varepsilon\left(R_{a} \cap R_{b}^{\circ}\right)=\varepsilon\left(R_{a} \star R_{b}^{\circ}\right)=\left\langle R_{b}^{\circ}, R_{a}^{\circ}\right\rangle=\overline{\left\langle R_{b}, R_{a}\right\rangle}=0,
$$

where we used above that for all $x, y \in H:\left\langle x^{\circ}, y^{\circ}\right\rangle=\overline{\langle x, y\rangle}$-see Observation 4.4, (1)-. Now, if $x \in R_{b}^{\circ}$ and $y \in R_{a}^{\circ}$, then $y^{\circ} \star_{\varphi} x \in R_{a} \cap R_{b}^{\circ}$ and $\langle x, y\rangle_{\varphi}=\varepsilon\left(y^{\circ} \star_{\varphi} x\right) \in \varepsilon\left(R_{a} \cap R_{b}^{\circ}\right)=\{0\}$.

Once that the above $\langle,\rangle_{\varphi}$-orthogonality is established, it is enough to show that $\langle x, x\rangle_{\varphi}>0$, for all $a$ and $\forall x \in R_{a}^{\circ}, x \neq 0$, and this is exactly the content of Lemma 6.5 -see also Observation 6.6-.

A proof in matricial terms of the above theorem, appears in $[13$, Theorem $2.8]$-see also the previous paper [3]-.

Corollary 6.8. If $H$ is a compact quantum group, then the automorphism $S^{2}: H \rightarrow H$ is positive definite with respect to the inner product defined by the integral $\varphi$.

Proof. The result follows from the equalities: $\left\langle S^{2}(x), x\right\rangle_{\varphi}=\varphi\left(S\left(x^{\circ}\right) S^{2}(x)\right)$ $=\varphi\left(S(x) x^{\circ}\right)=\left\langle x^{\circ}, x^{\circ}\right\rangle_{\varphi}$.

We summarize the above results in the following theorem (see [4, Proposition 2.4]).

Theorem 6.9. A o-Hopf algebra $H$ is a compact quantum group if and only if it is cosemisimple and the normal integral $\varphi: H \rightarrow \mathbb{C}$ is positive definite in the sense that $\varphi\left(S\left(x^{\circ}\right) x\right)>0$ for all $0 \neq x \in H$.

\section{The conjugacy of compact involutions}

In this section we present a detailed proof -using the methods introduced above- of a result that appeared in [4, Theorem 2.6]: if a cosemisimple Hopf algebra has two different compact o-structures, then they are conjugate by a positive Hopf algebra automorphism.

The proof is self-contained and should be thought as an expansion and elaboration of the one appearing in [4]. In the same manner than the original proof, ours is based in a few simple facts about positive linear transformations on finite dimensional complex vector spaces -and locally finite algebras- equipped with an inner product.

We recall some of the needed elementary results below.

Observation 7.1. Let $V$ be a vector space equipped with an inner product [, ] and $N: V \rightarrow V$ be a positive linear transformation. Assume moreover that $V$ is $N$-locally finite in the sense that $V$ is the direct sum of $N$ stable finite dimensional spaces. It is well known that if we work on the 
finite dimensional $N$-stable pieces of $V$, we can find positive real numbers $\lambda_{i}: i=1, \cdots, k$ and orthogonal projections $E_{i}: i=1, \cdots, k$ such that $N=\lambda_{1} E_{1} \oplus \cdots \oplus \lambda_{k} E_{k}$. The operator $P=\left(\lambda_{1}\right)^{1 / 2} E_{1} \oplus \cdots \oplus\left(\lambda_{k}\right)^{1 / 2} E_{k}$ is the only positive operator with respect to [, ] satisfying that $P^{2}=N$. The inverses of $N$ and $P$ are also positive operators with respect to [ , ]. All this can be globalized to all of $V$ in the obvious manner.

Moreover, if there exists a linear involution $\sigma: V \rightarrow V$ such that $\sigma N=$ $N^{-1} \sigma$, then $\sigma P=P^{-1} \sigma$, and this can be proved locally. In that situation, if $\lambda$ is an eigenvalue of $N$ and $E_{\lambda}$ is the corresponding eigenspace, then from the equality $\sigma N \sigma=N^{-1}$ we deduce that $\sigma\left(E_{\lambda}\right)=E_{\lambda^{-1}}$. Then, $\left.P \sigma\right|_{E_{\lambda}}=\left.(\sqrt{\lambda})^{-1} \sigma\right|_{E_{\lambda}}$ and thus, for $y \in E_{\lambda}, \sigma P \sigma y=(\sqrt{\lambda})^{-1} y=P^{-1} y$.

Observation 7.2. Here -for later use- we present two applications of the above construction, one to algebras and the other to simple coalgebras.

(1) Assume that additionally to the situation above we have that $V=$ $A$ is a $\mathbb{C}$-algebra equipped with an inner product $[$,$] and that$ $N: A \rightarrow A$ is a positive algebra automorphism. Using the fact that $N$ and $P$ have the same eigenspaces, it is easy to prove that $P$ is an algebra automorphism. Clearly, from $N(1)=1$ we deduce that $1 \in H$ is an eigenvector and then $P(1)=1$. Moreover, it is easy -and enough- to check the multiplicativity for a pair $x, y$ of eigenvectors of $P$-or equivalently, eigenvectors of $N-$. As $x y$ is also an eigenvector of $N$-and then of $P$-, the multiplicativity follows immediately.

(2) Later we will use also a dual version of the above result. If $N$ is a positive multiplicative coalgebra automorphism in the same situation that above, then its positive square root is also a coalgebra automorphism.

(3) If $C$ is an arbitrary coalgebra, it is clear that a linear map $W$ : $C \rightarrow C$ is a morphism of right comodules if and only if there exists a functional $\omega: C \rightarrow \mathbb{C}$ with the property that $W=w \star$ id. In that case $\omega=\varepsilon W$. Assume moreover that $C$ is a simple coalgebra and that $W$ is positive with respect to a given inner product $\langle$, on $C$. We want to prove that in this situation there exists another functional $\rho: C \rightarrow \mathbb{C}$ such that: $\rho^{2}=\omega$ and the map $\rho \star$ id $: C \rightarrow C$ is positive. Indeed, if we write the decomposition of $C$ as a sum of $W$-eigenspaces: $C=\bigoplus E_{a}$, then $\left.W\right|_{E_{a}}=\lambda_{a}$ id with $\lambda_{a}$ a positive real number. In that case $\left.\omega\right|_{E_{a}}=\lambda_{a} \varepsilon$. If we call $P$ the positive square root of $W$, it is clear that on each $E_{a}$, the operator $P$ is of the form $\sqrt{\lambda_{a}}$ id. From this expression it follows that $P$ is also a morphism of right comodules. To prove this we take $x \in E_{a}$ and hence, $P(x)=\sqrt{\lambda_{a}} x$ and $W(x)=\lambda_{a} x$. As $W$ is a morphism of right comodules, we deduce that if we write $\Delta(x)=\sum u_{i} \otimes v_{i}$ with 
vectors $v_{i}$ linearly independent, then $\lambda_{a} \sum u_{i} \otimes v_{i}=\sum W\left(u_{i}\right) \otimes v_{i}$. Hence, $W\left(u_{i}\right)=\lambda_{a} u_{i}$-i.e. $\Delta\left(E_{a}\right) \subset E_{a} \otimes C$ - and by the definition of $P$, we know that on the eigenvectors $u_{i}, P\left(u_{i}\right)=\sqrt{\lambda_{a}} u_{i}$. Hence $P$ is a morphism of right comodules and as such $P=\rho \star$ id for a certain linear functional $\rho: C \rightarrow \mathbb{C}$. In this situation it is clear that $\rho^{2}=\rho \star \rho=\omega$.

(4) Observe that we have proved above in part (3), that if $V$ is a right $C$-comodule, $W: V \rightarrow V$ is a morphism of $C$-comodules and $E$ is an eigenspace of $W$, then $E \subset V$ is a $C$-subcomodule. There is also a version of this result for left $C$-comodules.

Theorem 7.3. Let $(H, \circ)$ be a compact quantum group and $\diamond: H \rightarrow H_{c}$ be an involution such that $(H, \diamond)$ is a o-Hopf algebra. Then:

(1) There exists a Hopf algebra automorphism $P$, positive with respect to the inner product associated to $\circ$, such that the involutions $\diamond$ and $P \circ P^{-1}$ commute with each other.

(2) If $(H, \diamond)$ is also a compact quantum group, then $\diamond=P \circ P^{-1}$.

Proof. (1) Consider the map $Q: H \rightarrow H$ defined by $Q=\diamond \circ$. Clearly, $Q^{-1}=\circ \diamond, Q$ is an automorphism of Hopf algebras -therefore it commutes with the antipode- and $\varphi Q=\varphi$ where $\varphi$ is the normal integral on $H$. Moreover, $Q \circ=\circ Q^{-1}$ and $\diamond Q=Q^{-1} \diamond$. First, we prove that $Q$ is selfadjoint with respect to the inner product associated to $\circ$. Indeed:

$$
\begin{aligned}
& \langle Q(x), y\rangle=\varphi\left(S\left(y^{\circ}\right) x^{\circ \diamond}\right)=\left(\varphi Q^{-1}\right)\left(S\left(y^{\circ}\right) x^{\circ \diamond}\right) \\
= & \varphi\left(S\left(y^{\circ \diamond}\right) x\right)=\varphi\left(S\left(Q(y)^{\circ}\right) x\right)=\langle x, Q(y)\rangle .
\end{aligned}
$$

Therefore, $Q^{2}$ is a positive automorphism of Hopf algebras and also $Q^{2} \circ=\circ Q^{-2}$ and $\diamond Q^{2}=Q^{-2} \diamond$.

$$
\text { Let } H_{\rho}^{\prime}= \begin{cases}H_{\rho} & \text { if } H_{\rho}^{\diamond}=H_{\rho} \\ H_{\rho} \oplus H_{\rho}^{\diamond} & \text { if not }\end{cases}
$$

Clearly, for each $\rho \in \widehat{H}, H_{\rho}^{\prime}$ is a $\diamond$-subcoalgebra of $H$ and $H=$ $\bigoplus_{\rho \in \bar{H}} H_{\rho}^{\prime}$, for some $\bar{H} \subseteq \widehat{H}$.

Then, $H_{\rho}^{\prime}$ is $Q^{2}$-invariant and finite dimensional and for each $\rho \in \bar{H}$ we can define the operator $P_{\rho}: H_{\rho}^{\prime} \rightarrow H_{\rho}^{\prime}$, that is the positive fourth-root of $\left.Q^{2}\right|_{H_{\rho}^{\prime}}$. We define the operator $P: H \rightarrow H$ as the sum of all these $P_{\rho}$. In this situation $P$ is invertible, positive and $P^{4}=Q^{2}$.

Now, by applying twice the Observation 7.1 we deduce that $P \circ=$ $\circ P^{-1}$ and $\diamond P=P^{-1} \diamond$. 
Then:

$$
\begin{aligned}
\diamond P \circ P^{-1} \diamond P \circ P^{-1} & =\diamond \circ P^{-2} \diamond P \circ P^{-1}= \\
\diamond \circ \diamond P^{3} \circ P^{-1} & =Q^{2} P^{-4}=\mathrm{id}_{H} .
\end{aligned}
$$

Hence, we conclude that $\diamond P \circ P^{-1}=\left(\diamond P \circ P^{-1}\right)^{-1}=P \circ P^{-1} \diamond$. (2) Assume now that $(H, \diamond)$ is also a compact quantum group and call $[$,$] the inner product associated to \diamond$, i.e. defined by $[x, y]=$ $\varphi\left(S\left(y^{\diamond}\right) x\right)$.

In this situation, if $x, y \in H$, we have that

$$
\begin{aligned}
& \left\langle Q^{-1} x, y\right\rangle=\varphi\left(S\left(y^{\circ}\right) Q^{-1} x\right)=\varphi\left(S^{-1}(y)^{\circ} Q^{-1} x\right)= \\
& =(\varphi Q)\left(S^{-1}(y)^{\circ} Q^{-1} x\right)=\varphi\left(S^{-1}(y)^{\diamond} x\right)=\varphi\left(S\left(y^{\diamond}\right) x\right)=[x, y] .
\end{aligned}
$$

Hence $Q^{-1}$ is positive on $(H,\langle\rangle$,$) and so is Q$.

Now, as both $P^{2}$ and $Q$ are positive square roots of $P^{4}=Q^{2}$, we deduce that $P^{2}=Q$ and:

$$
P \circ P^{-1}=\circ P^{-2}=\diamond \diamond \circ P^{-2}=\diamond Q P^{-2}=\diamond
$$

and the proof is finished.

Observation 7.4. The general problem of the existence of a compact involution for a cosemisimple Hopf algebra is -in the knowledge of the authorswide open.

Due to well known results on semisimple Hopf algebras -see for example [18]-, in the case that the original Hopf algebra $H$ is finite dimensional, a compact finite quantum group is simply a (finite dimensional) semisimple Hopf algebra (with a normal left and right integral that we call $\varphi: H \rightarrow \mathbb{C}$ ) with the property that the sesquilinear form in $H$ defined by the formula: $\langle x, y\rangle=\varphi\left(S\left(y^{\circ}\right) x\right)$, is positive definite.

In the survey article [5], the following Question 7.8 is raised.

Given a semisimple Hopf algebra H, does it admit a compact involution?

As far as the authors are aware of, the answer to this question is not known.

Regarding this point, the particular case of abelian extensions of Hopf algebras is considered in [17]. In particular when the original Hopf algebras are of the form $H=\mathbb{C} F$ and $K=\mathbb{C}^{G}$, the author briefly considers the extension problem for $H$ and $K$ considered as finite quantum groups endowed with their natural o-structures. He proves that the group of extensions of $H$ and $K$ as o-Hopf algebras coincides with the group of extensions as Hopf algebras ([17, Remark 1.11, (2)]). 
One expects that this observation could be generalized to the case of arbitrary finite dimensional (abelian) extensions.

In the case of a not necessarily finite dimensional (cosemisimple) Hopf algebra $H$, the classical situation could be an indication of the results to be expected. At most one should expect to construct a compact involution in a sufficiently large quotient of $H$.

\section{The mien of the antipode of a compact quantum group}

In this section we intend to obtain a better understanding of the antipode antiautomorphism $S$ in the case of a compact quantum group, and for this purpose we apply in this particular set up, some of the standard tools in the theory of coFrobenius Hopf algebras: the modular function, the Nakayama automorphism, Radford's formula, etc. Our algebraic methods differ from the more standard analytical methods applied for example in [16].

We start by defining the positive antipode. Once we know (see Corollary 6.8) that $S^{2}$ is a positive Hopf algebra automorphism with respect to the inner product $\langle$,$\rangle given by the normal integral, it follows from the results$ mentioned in Observation 7.2 that there exists a positive automorphism of algebras and coalgebras that is the positive square root of $S^{2}$.

Definition 8.1. Let $H$ be a compact quantum group with integral $\varphi$ and associated inner product $\langle$,$\rangle . Then the unique positive automorphism of$ algebras and coalgebras $S_{+}: H \rightarrow H$ that satisfies

$$
S^{2}=S_{+}^{2} \text {. }
$$

is called the positive antipode of $H$.

Observation 8.2. It is clear that $S_{+}$leaves all the simple components $H_{\rho}$ invariant.

Our next objective is to prove that $S_{+}$is an inner automorphism and then to deduce that $S^{2}: H \rightarrow H$ is also an inner automorphism -see [16] for a proof of this result-.

This result will follow easily from the following well known fact: a coalgebra automorhism of a matrix coalgebra is inner -the dual of this result is attributed to A.A. Albert in a more general version-.

Theorem 8.3 (A.A.Albert). Let $V$ be a finite dimensional vector space and consider the coalgebra $c(V)$. Let $T: c(V) \rightarrow c(V)$ be an automorphism of coalgebras. Then, there exists a convolution invertible functional $\tau$ : $c(V) \rightarrow \mathbb{C}$, such that $T=(\tau \star \mathrm{id})\left(\mathrm{id} \star \tau^{-1}\right)$. Moreover, the linear functional $\tau$ is unique up to multiplication by a non zero scalar. 
Observation 8.4. Assume that $C$ is a finite-dimensional coalgebra and that $T: C \rightarrow C$ is a linear map that can be written as: $T=(\tau \star$ id $)($ id $\star \rho)$ and that satisfies the equality $\varepsilon T=\varepsilon$. Then $\rho=\tau^{-1}, \tau T=\tau, \rho T=\rho$ and $T$ is a morphism of coalgebras.

The following consequences of Albert's theorem 8.3, will be used later.

Corollary 8.5. Assume that $C$ is a $\circ$-coalgebra that is simple and equipped with a unitary inner product $\langle$,$\rangle . Let T$ be an automorphism of coalgebras and suppose that it can be decomposed as $T=(\tau \star \mathrm{id})\left(\mathrm{id} \star \tau^{-1}\right)$ where $\tau \star \mathrm{id}$ as well as $\mathrm{id} \star \tau^{-1}$ are selfadjoint. If $T$ is positive then $\tau \star \mathrm{id}$ and $\mathrm{id} \star \tau^{-1}$ can be taken to be positive.

Proof. Notice that the existence of a functional $\tau$ yielding a decomposition as above follows easily from Theorem 8.3. The selfadjointness has to be assumed as hypothesis.

Let us call $E_{\lambda}$ and $F_{\mu}$ the eigenspaces for $\tau \star$ id and id $\star \tau^{-1}$ corresponding to the eigenvalues $\lambda$ and $\mu$ respectively.

As $E=E_{\lambda} \cap F_{\mu}$ is a common eigenspace for the -commuting-operators $\tau \star$ id and id $\star \tau^{-1}$, it is clear that $\lambda \mu$ is an eigenvalue of $T$ and then it is positive and we deduce that $\lambda$ and $\mu$-are real and-have the same sign.

Next we prove that one of the subspaces $E_{+}=\bigoplus_{\lambda>0, \mu>0} E_{\lambda} \cap F_{\mu}$ or $E_{-}=\bigoplus_{\lambda<0, \mu<0} E_{\lambda} \cap F_{\mu}$ has to be trivial. Observe that they satisfy that $C=E_{+} \oplus E_{-}$.

It follows from Observation 7.2 part (4) that $\Delta\left(E_{\lambda}\right) \subset E_{\lambda} \otimes C$ and similarly $\Delta\left(F_{\mu}\right) \subset C \otimes F_{\mu}$. Then $\Delta\left(E_{\lambda} \cap F_{\mu}\right) \subset\left(E_{\lambda} \otimes C\right) \cap\left(C \otimes F_{\mu}\right)=E_{\lambda} \otimes F_{\mu}$. It is clear that for $\lambda>0, \mu>0, E_{\lambda}$ and $F_{\mu} \subset E_{+}$-and similarly for negative eigenvalues- and then it follows that: $\Delta\left(E_{+}\right) \subset E_{+} \otimes E_{+}, \Delta\left(E_{-}\right) \subset$ $E_{-} \otimes E_{-}$. As the coalgebra $C$ is simple, we deduce that one of these subspaces (subcoalgebras) has to be trivial.

Hence, after eventually changing $\tau$ by $-\tau$ we deduce that we can assume that the functional appearing in the formula $T=(\tau \star$ id $)\left(\mathrm{id} \star \tau^{-1}\right)$ has the property that $\tau \star \mathrm{id}$ and $\mathrm{id} \star \tau^{-1}$ are positive.

Lemma 8.6. Assume that $H$ is a compact quantum group and that $\langle$, is the inner product associated to the normal integral $\varphi$. For $\tau \in H^{*}$, the adjoints of the maps $\mathrm{id} \star \tau, \tau \star \mathrm{id}: H \rightarrow H$ with respect to the inner product are: $(\mathrm{id} \star \tau)^{*}=\mathrm{id} \star(\bar{\tau} \circ)$ and $(\tau \star \mathrm{id})^{*}=\left(\bar{\tau} S^{2} \circ\right) \star \mathrm{id}$ respectively.

Proof. We only prove the second assertion, the first is similar. We take $z, w \in H$ and compute explicitly $\langle(\tau \star \mathrm{id})(z), w\rangle$ and $\left\langle z,\left(\left(\bar{\tau} S^{2} \circ\right) \star \mathrm{id}\right)(w)\right\rangle$. 
In this situation the first expression is : $\langle(\tau \star \mathrm{id})(z), w\rangle=\tau\left(\sum z_{1}\left\langle z_{2}, w\right\rangle\right)=$ $\tau\left(\sum S^{2}\left(w_{1}^{\circ}\right)\left\langle z, w_{2}\right\rangle\right)=\left\langle z, \sum \bar{\tau}\left(S^{2}\left(w_{1}^{\circ}\right)\right) w_{2}\right\rangle=\left\langle z,\left(\left(\bar{\tau} S^{2} \circ\right) \star\right.\right.$ id $\left.)(w)\right\rangle$. The second, in the chain of equalities above, comes from Observation 6.3, part (4), equation (8).

We use the structure theorem -see Theorem 4.3 and also Observation 6.3- to conclude that whenever $H$ is a compact quantum group, the maps $S_{+}: H \rightarrow H$ and $S^{2}: H \rightarrow H$ are inner automorphisms.

Theorem 8.7. Let $H$ be a compact quantum group with antipode $S$ and let $S_{+}$be its positive antipode. Then, there exists a linear functional $\beta: H \rightarrow \mathbb{C}$ such that $S_{+}=(\beta \star \mathrm{id})\left(\mathrm{id} \star \beta^{-1}\right)$ and $S^{2}=\left(\beta^{2} \star \mathrm{id}\right)\left(\mathrm{id} \star \beta^{-2}\right)$.

Moreover, the restriction of $\beta$ to each simple component $H_{\rho}$, is unique up to multiplication by a non-zero scalar.

Furthermore, we can assume that the linear functional $\beta$ has been chosen as to satisfy the following additional properties:

(i) $\beta=\beta S_{+}, \beta^{-1}=\beta^{-1} S_{+}$;

(ii) $\beta=\bar{\beta}$ 。;

(iii) In the decomposition of the positive antipode $S_{+}=(\beta \star \mathrm{id})\left(\mathrm{id} \star \beta^{-1}\right)$, the operators $\beta \star \mathrm{id}$ and id $\star \beta^{-1}$ are selfadjoint, commute with each other and leave invariat the simple components $H_{\rho}$ of $H$.

(iv) The operators $\beta \star \mathrm{id}$ as well as $\mathrm{id} \star \beta^{-1}$ are positive.

Proof. We decompose $H=\bigoplus_{\rho} H_{\rho}$, where each $H_{\rho}$ is a simple coalgebra closed by the o operator of $H$. It was already proved that $S^{2}\left(H_{\rho}\right)=H_{\rho}$ and $S_{+}\left(H_{\rho}\right)=H_{\rho}$, hence for some $\beta_{\rho}: H_{\rho} \rightarrow \mathbb{C}$ the automorphism $\left.S_{+}\right|_{H_{\rho}}=$ $\left(\beta_{\rho} \star \mathrm{id}\right)\left(\mathrm{id} \star \beta_{\rho}^{-1}\right)$. If we call $\beta: H \rightarrow \mathbb{C}$ the direct sum of all the $\beta_{\rho}$, we have that $S_{+}=(\beta \star \operatorname{id})\left(\operatorname{id} \star \beta^{-1}\right)$.

For the rest of the proof we proceed as follows.

(i) Applying $\beta$ or $\beta^{-1}$ to the equality $S_{+}(x)=\sum \beta\left(x_{1}\right) x_{2} \beta^{-1}\left(x_{3}\right)$ we deduce the result.

(ii) Taking the adjoint of the operators in the formula $S_{+}=(\beta \star$ id $)($ id $\star$ $\left.\beta^{-1}\right)$ and recalling that $S_{+}$is selfadjoint we obtain the following: $S_{+}=(\beta \star$ $\mathrm{id})^{*}\left(\mathrm{id} \star \beta^{-1}\right)^{*}=\left(\left(\bar{\beta} S^{2} \circ\right) \star \mathrm{id}\right)\left(\mathrm{id} \star\left(\overline{\beta^{-1}} \circ\right)\right)=((\bar{\beta} \circ) \star \mathrm{id})\left(\mathrm{id} \star(\bar{\beta} \circ)^{-1}\right)$. Hence using again the uniqueness of $\beta$ up to a scalar in each simple coalgebra, we conclude that if we call $\beta_{\rho}$ the corresponding restriction, for each $\rho$ there exists a scalar $a_{\rho} \in \mathbb{C}: \beta_{\rho}=a_{\rho} \overline{\beta_{\rho}}$. It is clear that $\left|a_{\rho}\right|=1$. Indeed, we first deduce that $\beta_{\rho} \circ=a_{\rho} \overline{\beta_{\rho}}$ and then -after conjugation and multiplication by $a_{\rho}$ - we obtain that $\beta_{\rho}=\left|a_{\rho}\right|^{2} \beta_{\rho}$, i.e. $\left|a_{\rho}\right|=1$. 
Hence if we take $b_{\rho}$ to be a solution of the equation $b_{\rho} a_{\rho}=\overline{b_{\rho}}$, and call $\gamma_{\rho}=b_{\rho} \beta_{\rho}$, the new functional $\gamma_{\rho}$ satisfies the condition $\gamma_{\rho}=\overline{\gamma_{\rho}}$. Hence, after changing $\beta_{\rho}$ by $\gamma_{\rho}$ whenever it is needed, we obtain a new functional $\beta$ -we call it $\beta$ instead of $\gamma$ to simplify notations - that satisfies that $\beta=\bar{\beta}$ 。.

(iii) The fact that the operators $\beta \star$ id and id $\star \beta^{-1}$ are selfadjoint is a direct consequence of (i) and (ii). Clearly they leave invariant the simple components $H_{\rho}$.

(iv) This positivity result follows immediately from Corollary 8.5. Notice that the eventual change of sign necessary to make the operators positive that we might have performed in accordance with the mentioned corollarydoes not affect the properties (i), (ii) and (iii).

Next we record for future use, some easy consequences of the results about Radford's formula for coFrobenius coalgebras that appeared in [6]. Previously, in [27, Theorem 5.6] and in the context of compact quantum groups, some of these topics were considered.

Observation 8.8. We say that $H$ is a coFrobenius Hopf algebra over $\mathbb{C}$ if there exists a non-zero right (or equivalently a non-zero left) integral that will be called $\varphi: H \rightarrow \mathbb{C}$. In this situation there are bijections $x \mapsto(\varphi<x): H \rightarrow H^{*, \text { rat }}$ and $x \mapsto(x \rightarrow \varphi): H \rightarrow H^{*, \text { rat }}$.

(1) Using the above isomorphisms, we can construct the Nakayama automorphism of algebras characterized by the equality $\varphi(x y)=$ $\varphi(y \mathcal{N}(x))$-for all $x, y \in H-$. The modular function of the coFrobenius Hopf algebra $H$, is defined as $\alpha=\varepsilon \mathcal{N}$. The distinghished group like element in $H$-called $g$-is the unique group like element in $H$ such that for all $x \in H: \sum x_{1} \varphi\left(x_{2}\right)=\varphi(x) g$. In [6] the authors prove the following formulæ:

$$
\mathcal{N}(x)=\sum \alpha\left(x_{1}\right) S^{-2}\left(x_{2}\right), S^{4}(x)=g\left(\sum \alpha\left(x_{1}\right) x_{2} \alpha^{-1}\left(x_{3}\right)\right) g^{-1}, x \in H,
$$

where $g \in H$ is the distinguished group like element in $H$.

Clearly $\alpha: H \rightarrow \mathbb{C}$ is an algebra homomorphism and $\alpha^{-1}=\alpha S$.

(2) It follows easily from the equation (12) that the inverse of $\mathcal{N}$ can be computed as:

$$
\mathcal{N}^{-1}(x)=\sum \alpha^{-1}\left(x_{1}\right) S^{2}\left(x_{2}\right) .
$$

Also, $\mathcal{N}$ satisfies the following formula:

$$
\Delta(\mathcal{N}(x))=\sum \mathcal{N}\left(x_{1}\right) \otimes S^{-2}\left(x_{2}\right)=\sum \mathcal{N}\left(x_{1}\right) \otimes\left(\alpha^{-1} \star \mathcal{N}\right)\left(x_{2}\right)
$$


In other words, the map $\mathcal{N}$ is a morphism of comodules with the structures displayed below:

$$
\mathcal{N}:(H, \Delta)=H \rightarrow\left(H,\left(\mathrm{id} \otimes S^{-2}\right) \Delta\right)=H_{S^{-2}} .
$$

(3) The Nakayama morphism is comultiplicative if and only if $\alpha=\varepsilon$ or equivalently if and only if $\mathcal{N}=S^{-2}$. Indeed, from the comultiplicativity of $\mathcal{N}$ and from equation (14) we deduce that for all $x \in H$, $\sum x_{1} \otimes S^{-2}\left(x_{2}\right)=\sum x_{1} \otimes \mathcal{N}\left(x_{2}\right)$. Then $\mathcal{N}=S^{-2}$ and composing with $\varepsilon$ we deduce that $\alpha=\varepsilon$. The converse is clear.

(4) If $H$ is also unimodular, we have that $\mathcal{N}^{-1}=S^{-1} \mathcal{N} S$.

Indeed:

$$
\begin{aligned}
& \varphi\left(\mathcal{N}^{-1}(x) y\right)=\varphi(y x)=\varphi(S x S y)=\varphi(S y \mathcal{N} S x)= \\
& =\varphi\left(\left(S^{-1} \mathcal{N} S\right)(x) y\right) .
\end{aligned}
$$

(5) In the situation that the Hopf algebra is unimodular, the distinguished group like element $g=1$ and we have that:

$$
\begin{gathered}
S^{4}=(\alpha \star \mathrm{id})\left(\mathrm{id} \star \alpha^{-1}\right) \\
\mathcal{N}=\alpha \star S^{-2}=S^{2} \star \alpha, \mathcal{N}^{-1}=\alpha^{-1} \star S^{2}=S^{-2} \star \alpha^{-1}
\end{gathered}
$$

It follows easily from the above formulæ that $S^{2} \mathcal{N}=\mathcal{N} S^{2}=\alpha \star$ id and the Nakayama automorphism commutes with $S^{2}$.

In the case of a compact quantum group more information can be obtained about the maps considered above.

Lemma 8.9. Let $H$ be a compact quantum group equipped with the inner product $\langle$,$\rangle given by the normal integral \varphi$. Then:

(1) The Nakayama automorphism $\mathcal{N}$ is a positive operator with respect to $\langle$,$\rangle . Moreover it preserves the decomposition in simple compo-$ nents $H_{\rho}$-see Theorem 4.3-.

(2) The Nakayama automorphism commutes with $\circ$.

(3) If $\alpha: H \rightarrow \mathbb{C}$ is the modular function, then $\alpha \star \mathrm{id}: H \rightarrow H$ is a positive operator with respect to the inner product $\langle$,$\rangle .$

Proof. (1) The fact that $\mathcal{N}$ preserves the simple components $H_{\rho}$ follows immediately from Equation (12). The following chain of equalities -applied for $y=x$ - proves the positivity of $\mathcal{N}$.

$$
\langle\mathcal{N} x, y\rangle=\varphi\left(S\left(y^{\circ}\right) \mathcal{N} x\right)=\varphi\left(x S\left(y^{\circ}\right)\right)=\left\langle S\left(y^{\circ}\right), S\left(x^{\circ}\right)\right\rangle .
$$

For the last equality we used that: $S \circ S \circ=$ id.

(2) The proof of this commutation property follows from the equalities below:

$$
\varphi\left(y \mathcal{N}\left(x^{\circ}\right)\right)=\varphi\left(x^{\circ} y\right)=\overline{\varphi\left(x y^{\circ}\right)}=\overline{\varphi\left(y^{\circ} \mathcal{N}(x)\right)}=\varphi\left(y \mathcal{N}(x)^{\circ}\right) .
$$


(3) This positivity result follows directly from the fact that $\alpha \star$ id is the composition of $\mathcal{N}$ and $S^{2}$ that are positive and commuting operators.

Definition 8.10. Let $H$ be a compact quantum group and call $\langle$,$\rangle the$ unitary inner product associated to the normal integral $\varphi$. We define the positive square root of Nakayama automorphism -and denote it as $\mathcal{P}$ - as the unique automorphism of algebras that is positive and satisfies that $\mathcal{P}^{2}=\mathcal{N}$.

Corollary 8.11. Let $H$ be a compact quantum group and call $\varphi$ its normal integral. In the notations above, if $\beta$ is defined as in Theorem 8.7, then $\beta: H \rightarrow \mathbb{C}$ can be taken also to be an algebra morphism that satisfies $\beta^{4}=\alpha$.

Proof. The functional $\beta$ that we constructed before satisfies: $S^{4}=\alpha \star$ $\mathrm{id} \star \alpha^{-1}=\beta^{4} \star \mathrm{id} \star \beta^{-4}$. After restricting to each simple component $H_{\rho}$ we use Theorem 8.3 to prove the existence of a non zero scalar $c_{\rho} \in \mathbb{C}$, with the property that $\left.\alpha\right|_{H_{\rho}}=\left.c_{\rho} \beta^{4}\right|_{H_{\rho}}$. It is clear that being the operators associated to $\left.\alpha\right|_{H_{\rho}}$ and to $\left.\beta\right|_{H_{\rho}}$ positive -see Lemma 8.9 and Theorem 8.7the scalar $c_{\rho}$ is in fact a positive real number. If we change for each $\rho$ the functional $\beta_{\rho}$ by the functional $c_{\rho}^{1 / 4} \beta_{\rho}$ where $c_{\rho}^{1 / 4}$ is the positive fourth root of $c_{\rho}$, we obtain that $\beta^{4}=\alpha$ and this change does not affect the positivity of $\beta$.

In order to prove the multiplicativity, we observe that in accordance to the previous constructions $\beta \star$ id is a positive operator with the property that $(\beta \star \text { id })^{4}=\alpha \star$ id. It follows from Observation 7.2 that the unique fourth root of the multiplicative map $\alpha \star$ id is also multiplicative. Hence, $\beta \star \mathrm{id}$ as well as $\beta=\varepsilon(\beta \star \mathrm{id})$ are multiplicative.

Observation 8.12. (1) Once we know that $\beta$ is a morphism of algebras it is clear that:

$$
\beta^{-1}=\beta S \quad \beta=\beta^{-1} S .
$$

(2) Below, we list the expressions of some of the maps considered above in terms of $\beta$.

$$
\begin{array}{cc}
\mathcal{N}=\beta^{2} \star \mathrm{id} \star \beta^{2} & \mathcal{N}^{-1}=\beta^{-2} \star \mathrm{id} \star \beta^{-2} \\
\mathcal{P}=\beta \star \mathrm{id} \star \beta & S_{+}=\beta \star \mathrm{id} \star \beta^{-1} \\
\mathcal{P} S_{+}=\beta^{2} \star \mathrm{id} & \mathcal{P}^{-1} S_{+}=\mathrm{id} \star \beta^{-2} .
\end{array}
$$

The formula for $\mathcal{N}$ follows directly from equation (16), and the formula for $\mathcal{P}$ follows from the fact that id $\star \beta$ and $\beta \star$ id are positive and from the uniqueness of the square root of the positive operator $\mathcal{N}$. 
Next we use the automorphism $\mathcal{N}$ to compute explicitly the adjoint of $S$ and obtain some preliminary consequences of the normality of the operator $S$. This initial result will be refined in Theorem 8.15.

Lemma 8.13. Let $H$ be a compact quantum group with normal integral $\varphi$ and associated inner product $\langle$,$\rangle .$

(1) The adjoint of the operator $S: H \rightarrow H$ is $S^{*}=S \mathcal{N}^{-1}=\mathcal{N} S=$ $S^{-1} \star \alpha^{-1}$.

(2) In the situation above, $S S^{*}=S^{2} \mathcal{N}^{-1}=\mathcal{N}^{-1} S^{2}=\mathrm{id} \star \alpha^{-1}$ and $S^{*} S=\mathcal{N} S^{2}=S^{2} \mathcal{N}=\alpha \star$ id

(3) The operator $S$ is normal if and only if $S^{4}=$ id and $\alpha^{2}=\varepsilon$.

Proof. (1) For $x, y \in H$ we have that: $\langle S x, y\rangle=\varphi((S \circ) y S x)=\varphi\left(x y^{\circ}\right)$. Moreover: $\left\langle x, S \mathcal{N}^{-1} y\right\rangle=\varphi\left(\left(S \circ S \mathcal{N}^{-1}\right)(y) x\right)=\varphi\left(\left(\circ \mathcal{N}^{-1}\right)(y) x\right)=$ $\overline{\varphi\left(\mathcal{N}^{-1}(y) x^{\circ}\right)}=\overline{\varphi\left(x^{\circ} y\right)}=\varphi\left(x y^{\circ}\right)$.

In the above chain of equalities we have used first that $S \circ S=\circ$, then that $\varphi \circ=\bar{\varphi}$, later the definition of $\mathcal{N}$ to change the order of the product inside $\varphi$ and then again that $\varphi \circ=\bar{\varphi}$, -see Observation $6.2-$.

(2) Clearly: $S S^{*}=S^{2} \mathcal{N}^{-1}=\mathcal{N}^{-1} S^{2}=\left(\alpha^{-1} \star \mathrm{id}\right) S^{4}=\mathrm{id} \star \alpha^{-1}$.

Also $S^{*} S=\mathcal{N} S^{2}=\alpha \star$ id.

(3) Then, $S^{*} S=S^{*} S$ if and only if id $\star \alpha^{-1}=\alpha \star$ id. Composing this equality with $\varepsilon$ we obtain that $\alpha^{2}=\varepsilon$. Hence, we deduce that id $\star \alpha=\alpha \star$ id and this obviously implies that $S^{4}=(\alpha \star$ id) $\left(\right.$ id $\left.\star \alpha^{-1}\right)=$ id. The converse follows easily.

Observation 8.14. Assume that we are in the situation considered above.

(1) It is clear that the operators $S S^{*}$ and $S^{*} S$ leave all the simple coalgebras $H_{\rho}$ stable. Moreover the positive square roots of $S S^{*}$ and $S^{*} S$ are respectively: $\mathcal{P}^{-1} S_{+}$and $\mathcal{P} S_{+}$. Then the right polar decomposition of $S$ is $S=U \mathcal{P} S_{+}$and the left polar decomposition is $S=\mathcal{P}^{-1} S_{+} U$, where $U$ is unitary and equal to: $U=S S_{+}^{-1} \mathcal{P}^{-1}=$ $S_{+}^{-1} \mathcal{P} S$. It is clear that the operator $U: H \rightarrow H$ is an antimultiplicative involution -i.e. $U^{2}=\mathrm{id}-$. In the literature the operator $U$ is sometimes called the unitary antipode.

(2) In the case that $U=\mathrm{id}$, it is clear that the antipode $S$, being equal to $S=\mathcal{P} S_{+}$is multiplicative. As it is also antimultiplicative and bijective, we conclude that the product of $H$ is commutative.

Theorem 8.15. Let $H$ be a compact quantum group and consider the inner product given by the normal integral. Then, the following properties are equivalent: 
(1) The compact quantum group $H$ is involutive: i.e., $S^{2}=\mathrm{id}$.

(2) The positive antipode is trivial: i.e., $S_{+}=\mathrm{id}$.

(3) The unitary antipode coincides with $S$ : i.e., $U=S$.

(4) The antipode $S$ has finite order.

(5) The antipode $S$ is a normal operator: i.e., $S$ commutes with $S^{*}$.

(6) The antipode $S$ is a selfadjoint operator.

(7) The integral $\varphi: H \rightarrow \mathbb{C}$ is central: $\varphi(x y)=\varphi(y x)$-see [27]-.

(8) The automorphism $\mathcal{N}=\mathrm{id}$.

Proof. As the only positive square root of the identity is the identity itself, (2) follows clearly from (1). If $S$ has finite order so does $S^{2}$ and also the positive automorphism $S_{+}$. Then, $S_{+}=$id. This shows that the conditions (1), (2) and (4) are equivalent. To prove that (1) implies (5) we observe that in the hypothesis of (1), $\beta$ can be taken to be $\beta=\varepsilon$ and hence we deduce from Lemma 8.13 that $S$ is normal. Moreover, if we assume that the antipode is normal, using Lemma 8.13 we deduce that it has finite order. We have proved the equivalence between (1), (2), (4) and (5). The equivalence bewteen (6) and (7) is also clear. We know from Lemma 8.13 that $S^{*}=S \mathcal{N}^{-1}$. Hence $S$ is selfadjoint if and only if $\mathcal{N}=$ id and this happens -by definition of $\mathcal{N}$ - if and only if $\varphi(x y)=\varphi(y x)$.

Now we prove that condition (2) implies condition (6). Indeed in this case, it follows that $\beta=\varepsilon$ and then -in accordance with equation (20)- we deduce that $\mathcal{N}=$ id.

We finish the proof by observing that condition (3) is equivalent to condition (2). Indeed, in the case that $S=U$ as we know that $U^{2}=\mathrm{id}$, we conclude that $S^{2}=\mathrm{id}$. Conversely, if $S_{+}=\mathrm{id}$, then condition (7) guarantees that $\mathcal{N}=$ id and then $\mathcal{P}=S_{+}=$id. Using the formula $U=S S_{+}^{-1} \mathcal{P}^{-1}$ we conclude that $U=S$-see Observation 8.14-.

Some additional informacion can be obtained about the unitary antipode and Nakayama morphism in terms of the adjoint of the antipode.

Theorem 8.16. Let $H$ be a compact quantum group and consider the inner product given by the normal integral. Then, the following properties are equivalent:

(1) The adjoint of the antipode $S^{*}$ is an antimorphism of coalgebras.

(2) The unitary antipode $U$ is a morphism of coalgebras.

(3) The Nakayama morphism $\mathcal{N}$ is a comultiplicative morphism.

(4) The functional $\alpha$ coincides with the counit.

(5) The Nakayama morphism $\mathcal{N}$ equals $S^{-2}$. 
Proof. Suppose (1) holds. Then $S S^{*}$ is a morphism of coalgebras and so is $\mathcal{P} S_{+}$. Using that $S=U \mathcal{P} S_{+}$, we get that $U$ is an antimorphism of coalgebras.

Suppose (2) holds. As $S=U \mathcal{P} S_{+}, S$ is an antimorphism of coalgebras and $S_{+}$is a morphism of coalgebras, we deduce that $\mathcal{P}$ and $\mathcal{N}=\mathcal{P}^{2}$ are morphisms of coalgebras and we have (3).

Suppose now that (3) holds. Then $\mathcal{P}, \mathcal{P} S_{+}$and $S S^{*}=\left(\mathcal{P} S_{+}\right)^{2}$ are comultiplicative. Then, $S^{*}$ is an antimorphism of coalgebras.

Suppose now that (3) holds. From the equality $\mathcal{N}=\alpha \star S^{-2}$ we conclude that $\alpha \star$ id is comultiplicative. In that case applying $\varepsilon \otimes \varepsilon$ to the equality $\sum \alpha\left(x_{1}\right) x_{2} \otimes \alpha\left(x_{3}\right) x_{4}=\sum \alpha\left(x_{1}\right) x_{2} \otimes x_{3}$ we deduce that $\alpha \star \alpha=\alpha$ and then $\alpha=\varepsilon$, and we deduce that condition (4) is satisfied.

It is clear that if $\alpha=\varepsilon$, then $\mathcal{N}=S^{-2}$ and then it is comultiplicative. In other words from condition (5) we easily deduce condition (3).

\section{The quantum special unitary group}

In this section we want to illustrate our constructions in the case of the group $\mathrm{SU}_{\mu}(2, \mathbb{C})$, viewed as a $\circ$-Hopf algebra rather than as it is usually presented in terms of a $\star$-operator.

Definition 9.1. Let $\mu \in \mathbb{C}$ be a non zero real number such that $|\mu|<1$. We call $\mathrm{SU}_{\mu}(2, \mathbb{C})=\mathbb{C}\left\langle\alpha, \gamma, \gamma^{\circ}, \widehat{\alpha}\right\rangle$ the non commutative algebra in the variables written above, subject to the following multiplicative relations.

$$
\begin{gathered}
\widehat{\alpha} \alpha-\mu \gamma^{\circ} \gamma=1 \quad \alpha \widehat{\alpha}-\mu^{3} \gamma \gamma^{\circ}=1 \\
\gamma^{\circ} \gamma=\gamma \gamma^{\circ} \\
\mu \gamma \alpha=\alpha \gamma \quad \mu \gamma^{\circ} \alpha=\alpha \gamma^{\circ} \\
\mu^{-1} \gamma \widehat{\alpha}=\widehat{\alpha} \gamma \quad \mu^{-1} \gamma^{\circ} \widehat{\alpha}=\widehat{\alpha} \gamma^{\circ}
\end{gathered}
$$

The $\circ$ operator is defined as being multiplicative, involutive, conjugate linear and taking on the generators $\alpha, \widehat{\alpha}$ the values:

$$
\alpha^{\circ}=\alpha \quad \widehat{\alpha}^{\circ}=\widehat{\alpha}
$$

The comultiplication and counit are defined as:

$$
\begin{aligned}
& \Delta(\alpha)=\alpha \otimes \alpha+\mu^{2} \gamma^{\circ} \otimes \gamma \quad \Delta(\widehat{\alpha})=\widehat{\alpha} \otimes \widehat{\alpha}+\mu^{2} \gamma \otimes \gamma^{\circ} \\
& \Delta(\gamma)=\gamma \otimes \alpha+\hat{\alpha} \otimes \gamma \quad \Delta\left(\gamma^{\circ}\right)=\alpha \otimes \gamma^{\circ}+\gamma^{\circ} \otimes \widehat{\alpha} \\
& \varepsilon(\alpha)=1 \quad \varepsilon(\widehat{\alpha})=1 \quad \varepsilon(\gamma)=0 \quad \varepsilon\left(\gamma^{\circ}\right)=0
\end{aligned}
$$


The linear map $S: \mathrm{SU}_{\mu}(2, \mathbb{C}) \rightarrow \mathrm{SU}_{\mu}(2, \mathbb{C})$ defined on the generators as:

$$
S(\alpha)=\widehat{\alpha} \quad S(\widehat{\alpha})=\alpha \quad S(\gamma)=-\mu \gamma \quad S\left(\gamma^{\circ}\right)=-\mu^{-1} \gamma^{\circ},
$$

and then extended antimultiplicatively to all $\mathrm{SU}_{\mu}(2, \mathbb{C})$ is the antipode of the above structure.

It is well known that $\mathrm{SU}_{\mu}(2, \mathbb{C})$ is a compact quantum group and as such it is equipped with an integral and an inner product for which we shall not provide an explicit expression. See [26] and [27] for a detailed study of this family of Hopf algebras from the $C^{*}$-algebra viewpoint, where explicit formulæ are presented.

A direct computation shows that the linear functional $\theta: \mathrm{SU}_{\mu}(2, \mathbb{C}) \rightarrow \mathbb{C}$ defined by the equality $S^{2}=\theta \star \mathrm{id} \star \theta S$ can be taken as:

$$
\theta(\alpha)=|\mu|^{-1} \quad \theta(\widehat{\alpha})=|\mu| \quad \theta(\gamma)=\theta\left(\gamma^{\circ}\right)=0,
$$

and then extended multiplicatively.

In this situation, if we consider $\beta: \mathrm{SU}_{\mu}(2, \mathbb{C}) \rightarrow \mathbb{C}$ defined as:

$$
\beta(\alpha)=|\mu|^{-1 / 2} \quad \beta(\widehat{\alpha})=|\mu|^{1 / 2} \quad \beta(\gamma)=\beta\left(\gamma^{\circ}\right)=0,
$$

we find that the positive antipode is given on the generators as:

$$
S_{+}(\alpha)=\alpha \quad S_{+}(\widehat{\alpha})=\widehat{\alpha} \quad S_{+}(\gamma)=|\mu| \gamma \quad S_{+}\left(\gamma^{\circ}\right)=|\mu|^{-1} \gamma^{\circ} .
$$

In this situation we obtain the following formulæ for the Nakayama automorphism $\mathcal{N}$ and for $\mathcal{P}$.

$$
\begin{array}{rlll}
\mathcal{N}(\alpha)=\mu^{-2} \alpha & \mathcal{N}(\widehat{\alpha})=\mu^{2} \widehat{\alpha} & \mathcal{N}(\gamma)=\gamma & \mathcal{N}\left(\gamma^{\circ}\right)=\gamma^{\circ} \\
\mathcal{P}(\alpha)=|\mu|^{-1} \alpha & \mathcal{P}(\widehat{\alpha})=|\mu| \widehat{\alpha} & \mathcal{P}(\gamma)=\gamma & \mathcal{P}\left(\gamma^{\circ}\right)=\gamma^{\circ}
\end{array}
$$

The unitary antipode $U$ can be computed by the following formulae:

$$
U(\alpha)=|\mu| \widehat{\alpha} \quad U(\widehat{\alpha})=|\mu|^{-1} \alpha \quad U(\gamma)=-\operatorname{sg}(\mu) \gamma \quad U\left(\gamma^{\circ}\right)=-\operatorname{sg}(\mu) \gamma^{\circ}
$$

\section{References}

[1] Abella, A. Cosemisimple coalgebras, Ann. Sci. Math. Québec, vol 30, no. 2, 2006, pp. 119-133.

[2] Abella, A. and Andruskiewitsch, N. Compact matrix quantum groups arising from Hermitian Yang-Baxter coalgebras, Comm. Algebra, vol. 30, 2002, no.7, pp. 3107-3142.

[3] Andruskiewitsch, N. Some exceptional compact matrix pseudogroups, Bull. Soc. math. France, 120, 1992, pp. 297-325.

[4] Andruskiewitsch, N. Compact involutions of semisimple quantum groups, Czech. J. of Phys., vol 44, 1994, pp. 963-972. 
[5] Andruskiewitsch, N. About finite dimensional Hopf algebras. Quantum symmetries in theoretical physics and mathematics (Bariloche, 2000), Contemp. Math., 294, pp 1-57, Amer. Math. Soc., Providence, RI , 2002.

[6] Beattie, M., Bulacu, D. and Torrecillas, B. Radford's $S^{4}$ formula for co-Frobenius Hopf algebras. J. Algebra, 307 , 1, (2007), pp. 330-342

[7] Borel, A. Essays in the history of Lie groups and algebraic groups. History of Mathematics, vol. 21, American Mathematical Society, Providence, RI. 2001.

[8] Dijkhuizen, M.S. and Koornwinder, T.H. CQG algebras: a direct algebraic approach to compact quantum groups. Lett. Math. Phys. vol. 32, 1994, pp. 315-330;

[9] Ferrer Santos, W. Fourier theory for coalgebras, bicointegrals and injectivity for bicomodules. In Bergen, J. et. al.(eds.) Hopf algebras Proceedings from the international conference, De Paul University, Chicago, IL. USA. Held during the 2001-2002 academic year. Marcel Dekker, Pure and applied mathematics, vol 237, New York, 2004.

[10] Guichardet, A. Groupes quantiques: Introduction au point de vue formel. InterÉditions/ CNRS Éditions. Savoirs Actuels. Paris, 1995.

[11] Hurwitz, A. Über die Erzengung der Invarianten durch Integration. Nach. Gött. Ges. Wissench, 1897, pp. 71-90.

[12] Klymik A.U. and Schmüdgen. Quantum Groups and their Representations. Texts and Monographs in Physics, Springer-Verlag, Berlin, 1997.

[13] Koortwinder, T. General Compact Quantum Groups, a Tutorial. arXiv:hepth/9401114v1, 24 Jan 1994.

[14] Larson, R. G. Characters of Hopf algebras J. Algebra, vol 17, 1971, pp. 352-368.

[15] Manin, Y. Quantum groups and noncommutative geometry, Université de Montréal Centre de Recherches Mathématiques, Montreal, 1988.

[16] Masuda, T., Nakagami, Y. and Woronowicz, S.L. A $C^{*}$-algebra framework for the quantum groups. Int. J. of Math. vol 14, 2003, pp. 903-1001.

[17] Masuoka, A. Hopf algebra extensions and cohomology. In Montgomery, S. and Schneider, H-J., (eds.) New directions in Hopf algebras. MSRI Publications. bf 43, Cambridge University Press, Cambridge, England, 2002.

[18] Schneider, H.-J. Lectures on Hopf Algebras, notes by S. Natale. Trabajos de Matemática, Vol. 31/95, 1995, FaMAF, Córdoba. Argentina.

[19] Schur, I. Neue Anwendungen der Integralrechnung auf Probleme der Invariantentheorie 1. Mitteilung, Sitzungsber. Preuss. Akad. Wiss. Phys.-Math. Kl. 1924, pp. 189-208; Ges. Abh., II, 440-459.

[20] Sweedler, M. Integrals for Hopf algebras. Annals of Math. (2), vol 89, 1969, pp. 323-335.

[21] Sweedler, M. Hopf algebras. New York: W.A. Benjamin, Inc. 1969.

[22] Takeuchi, M. Survey of braided Hopf algebras. In Andruskiewitsch, N. et. al.(eds.) New trends in Hopf algebra theory Proceedings of the Colloquium on Quantum groups and Hopf algebras, La Falda, Sierras de Córdoba, Argentina. August 9-13, 1999. American Mathematical Society, Contemporary mathematics, 267. Rhode Island, USA. 2000.

[23] Weyl, H. Das gruppentheoretische Fundament der Tensorrechnung. Nachr. Gess. Wiss. Götteningen, Math.-Phys. Kl. 1924, pp. 218-224; G. A. II, 461-467.

[24] Weyl, H. Zur Theorie der Darstellung der einfachen kontinuierlichen Gruppen (Aus einem Scheriben an Herrn I. Schur). Sitzungsber. Preuss. Akad. Wiss. Berlin Phys.-Math. Kl. 1924, pp. 338-345; G. A. II, 453-460.

[25] Weyl, H. Theorie der Darstellung kontinuierlicher Gruppen durch lineare Transformatinen, I, II, III und Nachtrag. Math. Zeitschr. 23, 1925, pp 271-309, 24, 1926, pp. 328-376, 377-395, 789-791; G. A. II, 543-647; SHW, 262-366. 
[26] Woronowicz, S.L. Twisted SU(2) group. An Example of a Non-Commutative Differential Calculus. Publ. RIMS, Kyoto Univ. Vol 23, 1987, pp 117-181.

[27] Woronowicz, S.L. Compact matrix pseudogroups, Comm. Math. Phys, 111, 1987, pp. 613-665. 